\title{
Revisited Wurtzite CdSe Synthesis : a Gateway for the Versatile Flash Synthesis of Multi-Shell Quantum Dots and Rods
}

\author{
Emile Drijvers, ${ }^{1,3}$ Jonathan De Roo, ${ }^{1,2}$ Krisztina Fehér, ${ }^{4}$ Zeger Hens ${ }^{1,3}$ and Tangi Aubert ${ }^{1,3}$ \\ ${ }^{1}$ Physics and Chemistry of Nanostructures (PCN), and ${ }^{2}$ Sol-gel Centre for Research on Inorganic Powders and Thin films Synthesis \\ (SCRiPTS), Department of Inorganic and Physical Chemistry, Ghent University, Krijgslaan 281-S3, 9000 Ghent, Belgium. \\ ${ }^{3}$ Center for Nano and Biophotonics (NB-Photonics), Ghent University, Sint-Pietersnieuwstraat 41, 9000 Ghent, Belgium. \\ ${ }^{4}$ NMR and Structure Analysis Unit, Department of Organic and Macromolecular Chemistry, Ghent University, Krijgslaan 281-S4, 9000 \\ Ghent, Belgium.
}

KEYWORDS: Semiconductor, nanocrystal, NMR, CdSe/CdS/ZnS, giant core-shell.

\begin{abstract}
Quantum dot core-shell structures are essential to superior optical properties. However, Cd-based quantum dots grown from wurtzite CdSe cores still suffer from irreproducibility due to gel formation during the core synthesis. Moreover, stateof-the-art shelling procedures often remain time consuming. We report here (1) a new procedure of the wurtzite CdSe quantum dot synthesis which eliminates the phosphonic anhydride contamination and (2) the improvement and generalization of the flash synthesis for the growth of highly luminescent multi-shell heterostructures. The high versatility of the flash procedure enables the epitaxial shelling of wurtzite CdSe by $\mathrm{CdS}, \mathrm{ZnS}$ and even $\mathrm{CdZnS}$ alloys, resulting in a significant enhancement of their photoluminescence efficiency. Thanks to a modification of the $\mathrm{CdSe} / \mathrm{CdS}$ dot-in-rods synthesis to prevent tip-to-tip connections, the flash procedure could also be extended to these anisotropic structures for the growth of multi-shell nanorods as well. The high-quality optical and stability properties of these multi-shell flash heterostructures are of great interest for applications ranging from lighting and displays to long term cell labeling.
\end{abstract}

Colloidal quantum dots (QDs) have emerged as versatile building blocks for a broad class of applications ranging from photonics to biomedicine. ${ }^{1-3}$ The quantum confinement of the excited charge carriers inside semiconductor QDs gives rise to unique size-tunable optical properties. However, surface defects and poor surface passivation with organic ligands can cause trap emission and non-radiative recombination which consequently leads to low photoluminescent quantum yields (PLQY). ${ }^{4}$ Furthermore, the undesired single-dot emission intermittency, or ON/OFF blinking, attributed to the nonradiative loss of electron-hole pairs through Auger recombination is often observed in semiconductor QDs. ${ }^{5}{ }^{6}$ Inorganic materials with larger bandgaps are therefore usually employed to enhance surface passivation and to better insulate the electron-hole pair. ${ }^{7,8}$

Core-shell structures, such as CdSe/CdS QDs, have been extensively investigated over the last two decades as they offer high photoluminescent quantum yields, narrow and symmetrical emission spectra. In recent years, these heterostructures have increasingly gained attention, with the development of synthetic procedures for the growth of very thick shells. These so-called "giant" core-shell QDs have shown superior optical properties, including high robustness, PLQYs approaching unity and suppressed blinking. These enhanced properties result in part from the large shell volumes but also from other structural characteristics such as alloyed interfaces and high crystallinity. ${ }^{9-13}$
In this context, we recently developed the flash synthesis, a method specifically designed for the controllable epitaxial growth of very thick CdS shells within a few minutes of reaction time. ${ }^{14}$ These flash QDs exhibit high crystallinity and state-of-the-art properties with narrow emission spectra and high PLQY. In addition, Raman spectroscopy revealed an alloyed core/shell interface, resulting in a considerably reduced blinking rate. Thanks to their high quality and robustness, flash QDs have already demonstrated their great potential for bioapplications, ${ }^{15}$ with lasting optical properties even once encapsulated in a biocompatible silica shell and transferred to water, or for their integration in photonic devices. ${ }^{16,17}$ In spite of this combination of synthetic convenience and high material quality, little efforts have been made to extend seeded-growth approaches beyond $\mathrm{CdS}$ shelling of core $\mathrm{CdSe}$ QDs. The flash synthesis of $\mathrm{ZnS}$ and $\mathrm{CdZnS}$ shells would, nevertheless, offer a gateway to further optimize the properties of Cd-based core/shell QDs in view of surface passivation, exciton confinement and overall robustness against environmental influences. ${ }^{18}$

Originally, this flash synthesis was an adaptation of the method reported by Carbone et al. for the synthesis of anisotropic CdSe/CdS dot-in-rods. ${ }^{19}$ These nanorods (NRs) constitute another class of interesting materials as they show polarized emission along their long axis, a property of particular interest for lighting and display applications. ${ }^{19-21}$ However, these NRs can suffer from tip-to-tip connections. These hamper their large scale ordering, a necessary step to take full advantage of their unique properties in, for example, display applications. ${ }^{22}$ 
Our early findings quickly indicated that these connections would be equally detrimental for the growth of a $\mathrm{ZnS}$ shell in a subsequent step.

In the past few years, one of the key parameter that allowed for the synthesis core-shell QDs and NRs with excellent optical properties has been the development of syntheses at elevated temperatures (typically over $300{ }^{\circ} \mathrm{C}$ ). ${ }^{11,12,14,19}$ One can indeed reasonably expect that this produces materials with higher crystallinity. Diroll et al. have notably reported that core-shell structures synthesized at higher temperature show optical properties with enhanced robustness and thermal stability. ${ }^{23}$ At those temperatures, only the wurtzite crystal structure is stable. Thus, since the synthesis of such core-shell structures relies on a seeded epitaxial growth, it therefore requires the synthesis of wurtzite $\mathrm{CdSe}$ QDs beforehand. As it happens, many of the above mentioned heterostructures start from the same wurtzite CdSe QD synthesis. ${ }^{11,12,14,19}$ This particular synthesis makes use of a phosphonic acid for the complexation of the cadmium precursor and stabilization of the QDs. However, the formation of a phosphonic anhydride, during this synthesis has already extensively been addressed. ${ }^{24-}$ 27 This by-product is rather insoluble at lower temperatures and its elimination still remains a challenge. Indeed, this phosphonic anhydride typically causes a gelification of the QD suspension, which is a strong limitation for their use in the subsequent synthesis of high quality core-shell QDs, as well as for the upscaled production of these QDs.

Here, we develop a generic, multiple flash approach for the formation of a diversity of Cd-based core/shell QDs and NRs. To achieve this, we first tweak the original wurtzite CdSe QDs synthesis to prevent the phosphonic anhydride formation by adding a long chain alcohol to the reaction. This reproducibly yields larger amounts of QDs with higher purity and we provide herein a detailed description of their novel surface chemistry through nuclear magnetic resonance (NMR) spectroscopy. From these cores, we introduce a standardized flash synthesis of high quality core-shell QDs. We show that the purity of the core materials is key to obtain core-shell QDs with optimal properties. This is evidenced through the synthesis of $\mathrm{CdSe} / \mathrm{CdS}$ QDs with enhanced optical properties. We thereafter demonstrate that the flash procedure can be conveniently extended to the growth of $\mathrm{ZnS}$ and alloyed CdZnS layers, resulting in multi-shell QDs with even higher PLQYs. In view of the synthesis of multi-shell NRs we also modified the dotin-rod synthesis in order to prevent the tip-to-tip connections observed between rods, simply by changing the length of the short-chained phosphonic acid. Thanks to this modification of the original dot-in-rod synthesis, we demonstrate how the standardized flash procedure can also be applied to these anisotropic structures for the synthesis of CdSe/CdS/ZnS NRs. We thus conclude that the standardization of this flash procedure presented here constitutes an essential step towards the large scale production of these materials, regardless of the grown material.

\section{EXPERIMENTAL SECTION}

Materials. $\mathrm{CdO}(\geq 99.99 \%)$, oleyl alcohol $(\mathrm{OlOH}, 85 \%)$ and $\mathrm{ZnO}(99.999 \%)$ were purchased from Sigma-Aldrich. Noctadecylphosphonic acid (ODPA, $\geq 97 \%$ ), ntetradecylphosphonic acid (TDPA, $\geq 97 \%$ ) and ndodecylphosphonic acid (DDPA, $\geq 97 \%$ ) were purchased from PlasmaChem GmbH. Trioctylphosphine (TOP, $\geq 97 \%$ ) and sulfur (S, 99.999\%) were purchased from Strem Chemicals. Trioctylphosphine oxide (TOPO, for synthesis) was purchased from Merck Millipore while selenium powder (Se, -200 mesh, 99.999\%) and oleic acid (OA, 90\%) from Alfa Aesar. Toluene, methanol and chloroform were purchased from Fiers. The stock solutions of TOP-Se $(1.7 \mathrm{M})$ and TOP-S $(2.4 \mathrm{M})$ were prepared by dissolving $1.343 \mathrm{~g}$ of the selenium powder and $0.7704 \mathrm{~g}$ of sulfur in $10 \mathrm{~mL}$ of TOP respectively. Toluene $\mathrm{d}^{8}$ ( $99.5 \%$ deuterated) was purchased from Euriso-top.

Synthesis of the wurtzite CdSe core QDs. This new wurtzite CdSe QDs synthesis was adapted from the previously reported procedure in literature.$^{19} 1.5 \mathrm{mmol}(0.1925 \mathrm{~g})$ of $\mathrm{CdO}, 6 \mathrm{mmol}$ $(1.670 \mathrm{~g})$ of n-tetradecylphosphonic acid (TDPA), $24 \mathrm{mmol}$ $(6.44 \mathrm{~g})$ of oleyl alcohol $(\mathrm{OlOH})$ and $10 \mathrm{~g}$ of trioctylphosphine oxide (TOPO) were mixed in a $50 \mathrm{~mL}$ three-neck flask. The mixture was heated to $150{ }^{\circ} \mathrm{C}$ under nitrogen atmosphere for one hour. The solution was then heated to $350{ }^{\circ} \mathrm{C} .2 \mathrm{~mL}$ of trioctylphosphine (TOP) was injected as soon as the solution became colorless, indicating the dissolution of $\mathrm{CdO}$. Once the mixture reached $350{ }^{\circ} \mathrm{C}, 2.55 \mathrm{mmol}(1.5 \mathrm{~mL})$ of a $1.7 \mathrm{M}$ trioctylphosphine selenide (TOP-Se) solution was injected. After a reaction time of typically few seconds to few tens of seconds depending on the envisaged QD size, the reaction was quenched by a sudden drop in temperature using a water bath pre-heated to $80^{\circ} \mathrm{C}$ to avoid cracking of the flask. Once the temperature of the mixture reached $80{ }^{\circ} \mathrm{C}, 20 \mathrm{~mL}$ of methanol was injected in the flask to precipitate the QDs. The mixture was then centrifuged at $4000 \mathrm{rpm}$ for 1 minute. The supernatant was discarded and the QDs were purified three time with toluene and methanol as solvent and non-solvent. The QDs were dispersed and stored in $3 \mathrm{~mL}$ toluene. Overnight, the possible and very limited solid formation was removed by centrifuging the sample. The size of the QDs was determined from the position of the first excitonic absorption peak using the sizing curve of Mulvaney et al. ${ }^{28}$ The concentration of the wurtzite CdSe dispersion was determined based on their absorbance at $300 \mathrm{~nm}$ and $350 \mathrm{~nm}$ in toluene using the corresponding intrinsic absorption coefficients of wurtzite CdSe $\left(\mu_{300}=244046 \mathrm{~cm}^{-1}\right.$ and $\left.\mu_{350}=130823 \mathrm{~cm}^{-1}\right)$ calculated from the Maxwell-Garnett effective medium theory. ${ }^{29,}{ }^{30}$ As in the original synthesis described by Carbone et al. ${ }^{19}$ the size of the CdSe QDs is adjusted by controlling the reaction time. Nevertheless, one should note that for QDs sizes below $3 \mathrm{~nm}$, reaction times of no more than a few seconds are required, which can be hard to achieve and with poor size reproducibility. Thus, in those case it is more efficient to use the volume of oleyl alcohol to quench the reaction, i.e. the oleyl alcohol is not initially present in the reaction flask but injected after the reaction time, which result in a very fast and reproducible quenching. In our experiments, this always led to the same results regarding the purifications of these QDs. In order to facilitate the comparison of their optical properties, all the QDs and NRs reported here were prepared starting from $3 \mathrm{~nm}$ CdSe cores, i.e. synthesized with the oleyl alcohol already present in the reaction flask.

Flash Synthesis of Multi-Shell Quantum Dots. The versatile flash synthesis can be applied for the growth of different shell materials, such as $\mathrm{CdS}, \mathrm{ZnS}$ and even alloyed $\mathrm{CdZnS}$. For all shell growths, $2.5 \mathrm{~g}$ of oleic acid (OA) and $5 \mathrm{~g}$ of TOPO were added to a three-neck flask together with $\mathrm{CdO}$ and/or $\mathrm{ZnO}$, for the respective growth of the desired material. The amounts of $\mathrm{CdO}$ and/or $\mathrm{ZnO}$ were calculated based on the envisaged shell thickness, considering a full anionic chemical yield, and with a 
molar excess of 1.2 of the cation compared to the anion. The thicknesses of the shells can hence be tuned by varying the amounts of $\mathrm{CdO}$ and/or $\mathrm{ZnO}$. The mixture was heated to 150 ${ }^{\circ} \mathrm{C}$ under nitrogen atmosphere for one hour. The solution was then heated to $350{ }^{\circ} \mathrm{C}$ where $1 \mathrm{~mL}$ of TOP was injected as soon as the solution became colorless, indicating the dissolution of $\mathrm{CdO}$ and/or $\mathrm{ZnO}$. After the temperature recovered, a solution was injected, containing the seed QDs (i.e. either $\mathrm{CdSe}$ or $\mathrm{CdSe} / \mathrm{CdS}$ QDs depending on the synthesis step), trioctylphosphine sulfide (TOP-S, $2.4 \mathrm{M}$ ) of which the volume was always adjusted to $2 \mathrm{~mL}$ with TOP, to account for the same temperature drop. The amount of seeds injected for the shell growth varied depending on the synthesis step. Typically, the first shell growth was executed on $200 \mathrm{nmol}$ of seeds while the subsequent shell growths were executed on $15 \mathrm{nmol}$ then on $5 \mathrm{nmol}$ of seeds. After 5 minutes, the reaction was quenched by a sudden drop in temperature using a pre-heated water bath. Once the mixture has cooled down to room temperature, the QDs were precipitated by adding $10 \mathrm{ml}$ of methanol and $2 \mathrm{ml}$ of toluene to ensure miscibility of the mixture. The QDs were collected by centrifugation at $4000 \mathrm{rpm}$ for 3 min and further purified twice with toluene and methanol as solvent and non-solvent. The QDs were dispersed and stored in toluene. After each shell growth, the size of the QDs was determined by TEM analyses. In the case of QDs with only $\mathrm{CdS}$ shells their concentration was determined based on their absorbance in toluene at $300 \mathrm{~nm}$ and $350 \mathrm{~nm}$ using the corresponding intrinsic absorption coefficients of the $\mathrm{CdSe} / \mathrm{CdS}$ calculated from the Maxwell-Garnett effective medium theory depending on the ratio between the CdSe core and $\mathrm{CdS}$ shell volumes. ${ }^{29,30}$ For QDs with either $\mathrm{ZnS}$ and/or $\mathrm{CdZnS}$ shells, there is not yet a consistent model to determine the intrinsic absorption coefficients of such complex heterostructures. In those cases, the concentrations were estimated from the amount of seeds involved in the reaction to which $10 \%$ were subtracted to account for the QDs lost in the reaction and purification steps.

Flash Synthesis of Multi-Shell Nanorods. Firstly, CdSe/CdS dot-in-rods were grown from the wurtzite CdSe seeds, following the method of Carbone et al., ${ }^{19}$ which was slightly modified for further improvement of these NRs. For all syntheses, 5 $\mathrm{g}$ of TOPO was added to a three-neck flask, with a varying amount of octadecylphosphonic acid (ODPA), dodecylphosphonic acid (DDPA) and $\mathrm{CdO}$, depending on the desired diameter and length of the NRs. The total amount of phosphonic acid to Cd molar ratio was of $3: 1$, while the molar ratio ODPA to DDPA was of $1.5: 1$. The mixture was heated to $150{ }^{\circ} \mathrm{C}$ under nitrogen atmosphere for one hour. The solution was then heated to $350{ }^{\circ} \mathrm{C}$ where $1 \mathrm{~mL}$ of TOP was injected as soon as the solution became colorless, indicating the dissolution of $\mathrm{CdO}$. After the temperature recovered, a solution was injected, containing the CdSe seeds, TOP-S $(2.4 \mathrm{M})$ and whose volume was always adjusted to $2 \mathrm{~mL}$ with TOP to account for the same temperature drop. In this case, the rods were grown from $50 \mathrm{nmol}$ of CdSe seeds. Similarly to the growth of CdS shells on spherical QDs, the molar ratio $\mathrm{Cd}$ to $\mathrm{S}$ was of 1.2:1 to ensure a cation rich surface. The reaction was quenched after 8 minutes using a pre-heated water bath. Precipitation of the NRs was achieved by injecting $5 \mathrm{ml}$ of toluene and $10 \mathrm{ml}$ of methanol when the mixture had reached $120^{\circ} \mathrm{C}$ and $80^{\circ} \mathrm{C}$ respectively. The NRs were then immediately collected by a short centrifugation step of $1 \mathrm{~min}$ at 4000 rpm. Longer centrifugation durations or a lower temperature of the mixture would typically result in the precipitation of a phosphonic anhydride polymer that would hardly be separable from the NRs and should therefore be avoided. The NRs were further purified twice with toluene and methanol as solvent and non-solvent. Finally the NRs were dispersed and stored in toluene. The growth of a $\mathrm{ZnS}$ shell on the $\mathrm{CdSe} / \mathrm{CdS}$ dot-inrods was performed with the same procedure described in the previous paragraph for spherical QDs, using typically $10 \mathrm{nmol}$ of NRs. The dimensions of the NRs were systematically determined by TEM observations. In the case of purely $\mathrm{CdSe} / \mathrm{CdS} \mathrm{NRs}$, the concentrations were determined from the absorbance at $300 \mathrm{~nm}$ and $350 \mathrm{~nm}$, by considering that only the very large volume of wurtzite $\mathrm{CdS}$ contributes to the absorption at these wavelengths, and using its intrinsic absorption coefficients $\left(\mu_{300}=173021 \mathrm{~cm}^{-1}\right.$ and $\left.\mu_{350}=118769 \mathrm{~cm}^{-1}\right)$ calculated from the Maxwell-Garnett effective medium theory, ${ }^{29,}{ }^{30}$ and considering the NRs as cylinders. In the case of NRs with a $\mathrm{ZnS}$ shell, the concentration were estimated from the amount of seeds involved in the reaction to which $10 \%$ were subtracted to account for the NRs lost in the reaction and purification steps.

Material characterization. Bright field transmission electron microscopy (TEM) images and energy-dispersive $\mathrm{X}$-ray spectroscopy (EDX) measurements were made using a Cscorrected JEOL 2200FS microscope. Absorption spectra were taken using a Perkin Elmer Lambda 950 spectrometer. Photoluminescence measurements were done on an Edinburgh Instruments FLSP920 UV-Vis-NIR spectrofluorimeter. For steady-state measurements, a $450 \mathrm{~W}$ xenon lamp was used as the excitation source. All steady-state emission spectra were recorded for an excitation wavelength of $365 \mathrm{~nm}$ and were corrected for the detector sensitivity. The PLQYs were measured by using an integrating sphere, with an improved method adapted from Mello et al. ${ }^{31}$ in order to correct the measurements for internal reflections. ${ }^{15}$ The time-resolved measurements were performed using a picosecond pulsed LED (331 $\mathrm{nm}$, bandwidth $9.4 \mathrm{~nm}$, pulse width $872 \mathrm{ps}$ ) as the excitation source, and collecting the signal at the maximum of emission (bandwidth $10 \mathrm{~nm}$ ). Lifetimes were calculated by fitting the first $90 \%$ of the photoluminescence (PL) decay traces with a single exponential function (Equation 1):

$$
R(t)=e^{(-t / \tau)}+A
$$

With $A$ being a constant to account for the long tail of some decays. Results of the fits always gave $\chi^{2}$ values below 1.15. Nuclear magnetic resonance (NMR) samples were prepared by drying the purified QDs dispersion with a nitrogen flow which were then redispersed in $500 \mu \mathrm{L}$ deuterated toluene and inserted in a $5 \mathrm{~mm}$ tube. The sample temperature was set to 298.15 K. NMR measurements were recorded on a Bruker Avance III spectrometer operating at a ${ }^{1} \mathrm{H}$ frequency of $500.13 \mathrm{MHz}$ and equipped with a BBI-Z probe. Quantitative ${ }^{1} \mathrm{H}$ spectra were recorded with a $30 \mathrm{~s}$ delay between scans to allow full relaxation of all NMR signals and the 1D spectra were multiplied with an exponential window function. The quantification was done by using the Digital ERETIC method. Diffusion measurements (2D DOSY) were performed using a doublestimulated echo sequence for convection compensation and with monopolar gradient pulses. ${ }^{32}$ Smoothed rectangle gradient pulse shapes were used throughout. The gradient strength was varied linearly from 2 to $95 \%$ of the probe's maximum value (calibrated at $50.2 \mathrm{G} / \mathrm{cm}$ ) in 64 steps, with the gradient pulse duration and diffusion delay optimized to ensure a final 
attenuation of the signal in the final increment of less than $10 \%$ relative to the first increment. The alkene and methyl proton resonances were corrected prior to integration by subtracting a linear background from the measured intensity. The diffusion coefficients were obtained by fitting the appropriate Stejskal-Tanner equation (Equation 2) to the signal intensity decay. ${ }^{33}$

$$
I=I_{0} e^{\left[-D \gamma^{2} g^{2} \delta^{2}(\Delta-0.6 \delta)\right]}
$$

Two-dimensional ${ }^{1} \mathrm{H}$ NOESY (nuclear Overhauser effect spectroscopy) spectra were acquired using a standard pulse sequence from the Bruker library, noesygpphpp. NOESY mixing time was set to $300 \mathrm{~ms}$ and with 4096 data points set in the direct dimension and 512 data points set in the indirect dimension. For 2D processing, before Fourier transformation, the 2D spectra were multiplied with a squared sine bell function in both dimensions. ${ }^{1} \mathrm{H}-{ }^{13} \mathrm{C}$ HMBC (Heteronuclear Multiple-Bond Correlation) and ${ }^{1} \mathrm{H}-{ }^{31} \mathrm{P}$ HMQC (Heteronuclear Multiple Quantum Correlation) spectra were acquired using standard pulse sequences from the Bruker library, hmbcgplpndqf and hmqcgpndqf respectively. The delays were set to long range ${ }^{n} \mathrm{~J}^{1}{ }_{\mathrm{H}}^{13}{ }_{\mathrm{C}}$ and ${ }^{n} \mathrm{~J}_{\mathrm{H}}^{1}{ }_{\mathrm{P}}^{31}$ couplings of $8 \mathrm{~Hz}$ and $12 \mathrm{~Hz}$ in the ${ }^{1} \mathrm{H}-{ }^{13} \mathrm{C} \mathrm{HMBC}$ and ${ }^{1} \mathrm{H}-{ }^{31} \mathrm{P}$ HMQC respectively, while the one bond ${ }^{1} \mathrm{~J}_{\mathrm{H}}^{1}{ }^{13} \mathrm{C}$ couplings of $145 \mathrm{~Hz}$ were filtered out in the ${ }^{1} \mathrm{H}-{ }^{13} \mathrm{C}$ HMBC.

\section{RESULTS AND DISCUSSION}

Revisited Synthesis of the Wurtzite CdSe Quantum Dots. Cadmium phosphonate precursors are usually used in the synthesis of wurtzite CdSe QDs. However, phosphonic acids are prone to polymerization ${ }^{24-27}$ and this leads to the precipitation of part of the QD dispersion after synthesis and/or during purification. This is illustrated in Figure 1a which shows an image of a partially solidified CdSe QD dispersion due to the presence of a insoluble phosphonic anhydride. This is a serious limitation which also compromises the chemical yield of the synthesis and points in general to a poorly controlled ligand shell and hence limited colloidal stability. Moreover, coreshell QDs grown from these solidified cores typically show poor structural morphology, see Figure 1b. We have found that for small scale reactions, some purification methods such as a precipitation and centrifugation at elevated temperatures or the repeated purification of the QDs with a short chain amine, could partially remove the phosphonic anhydride. However, these techniques do not show satisfying results for larger scale reactions. Although problematic, the phosphonate precursor seems difficult to replace considering the high temperatures (over $300{ }^{\circ} \mathrm{C}$ ) that are required to ensure a high quality wurtzite structure. For instance, using a cadmium oleate precursor at those temperatures results in reactions too fast to control, yielding QDs with diameters persistently exceeding $5 \mathrm{~nm}$.

In chemical terms, the issue with the wurtzite CdSe QDs synthesis appears well understood. The excess phosphonic acid and cadmium phosphonate in the reaction mixture can readily react to form a phosphonic anhydride polymer, Figure 2c. This anhydride polymer precipitates with the nanocrystals during centrifugation, compromising the purification of the QDs. There is a possibility that the tightly bound phosphonate also reacts via its remaining free hydroxyl group to form the anhydride.
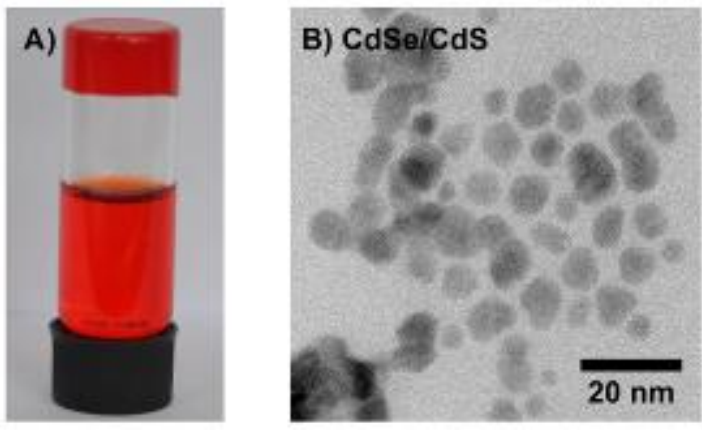

Figure 1. a) Image of an upside down vial containing wurtzite CdSe QDs synthesized with the method described in the literature, ${ }^{19}$ with the solidified fraction still attached to the bottom of the vial. b) TEM image of core-shell QDs grown from the solidified CdSe cores which result in particles with poor structural morphology.

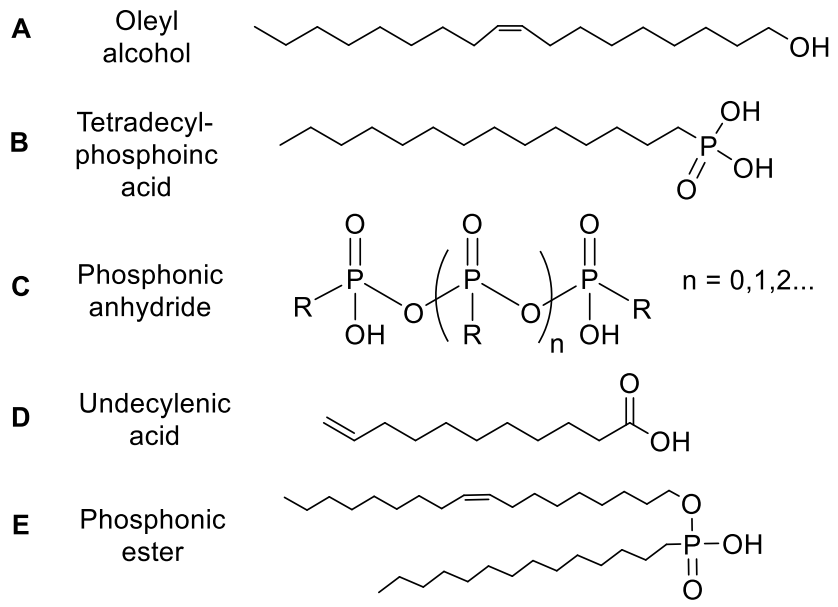

Figure 2. Molecular structures of a) oleyl alcohol $(\mathrm{OlOH}), \mathrm{b})$ tetradecylphosphonic acid (TDPA), c) a phosphonic anhydride, d) undecylenic acid and e) a phosphonic ester.

Use of oleyl alcohol (OlOH). In order to prevent the formation of this phosphonic anhydride, we decided to use a mixture of $\mathrm{OlOH}$ and phosphonic acid to dissolve the $\mathrm{CdO}$ and thus prepare the actual cadmium precursor. By this approach, the alcohol and the phosphonic acid form a phosphonic ester, which only contains one hydroxyl group, see Figure 2e. By removing one hydroxyl group through ester formation, this strategy avoids the condensation of the phosphonic anhydride polymer, which seems to be confirmed by the lack of precipitates or gels upon purification of the QDs using classical solvent-nonsolvent procedures. Up to now, octadecylphosphonic acid (ODPA) was the phosphonic acid of choice in the wurtzite $\mathrm{CdSe}$ synthesis. Here, we substituted it for tetradecylphosphonic (TPDA) as the resulting phosphonic ester between ODPA and $\mathrm{OlOH}$ proved to be insoluble, opposite from the respective TDPA ester.

Solution ${ }^{1} \mathrm{H}$ and ${ }^{31} \mathrm{P}$ NMR analysis was subsequently employed to characterize the surface of the wurtzite CdSe QDs and confirm the presumed role of $\mathrm{OlOH}$. According to the sole occurrence of a broadened $\mathrm{CH}_{3}$ resonance (Figure 3a, around $1 \mathrm{ppm})$, the dispersion is sufficiently purified and only bound ligands are present. ${ }^{34}$ However, both TDPA and $\mathrm{OlOH}$ have a $\mathrm{CH}_{3}$ resonance, rendering it impossible to distinguish both species by this resonance. In contrast, only $\mathrm{OlOH}$ has an alkene resonance at $5.5 \mathrm{ppm}$, which makes it easily identifiable 
in the ${ }^{1} \mathrm{H}$ NMR spectrum. In addition, this resonance is broadened, confirming the interaction of $\mathrm{OlOH}$ with the CdSe surface. Diffusion NMR (DOSY) measurements were carried out and by fitting the diffusion decay to the appropriate StejskalTanner equation, ${ }^{33}$ the diffusion coefficient was determined (Figure 3b). For both the $\mathrm{CH}_{3}$ and the alkene resonance, we find a diffusion coefficient of $105 \mu \mathrm{m}^{2} / \mathrm{s}$, which is converted by the Stokes-Einstein equation to a solvodynamic diameter of $7 \mathrm{~nm}$. This size is consistent with a nanocrystal size of $3 \mathrm{~nm}-$ as determined from the wurtzite CdSe sizing curve - and an organic capping layer of $2 \mathrm{~nm}$. This shows that both $\mathrm{OlOH}$ and TDPA diffuse together with the QDs and are thus tightly bound to the QD surface. Although this feat is not unsurprising for a strong X-type ligand as TDPA, it is unlikely for $\mathrm{OlOH}$ since alcohols were found very weak L-type ligands, typically exchanging fast between a free and a bound state. ${ }^{35}$ Therefore, we propose that $\mathrm{OlOH}$ has formed a phosphonic ester with TDPA (Figure 2e), which is bound to the CdSe surface in its deprotonated form.

To support these conjectures, we sought to strip the surface ligands from the surface. Although carboxylic acids are too weak an acid to displace phosphonic acids, ${ }^{36}$ it was shown before that amines can promote the exchange of a stronger acid for a weaker one. ${ }^{37-39}$ Therefore, we added a large excess of undecylenic acid and butylamine to the QD dispersion and analyzed the exchanged species in-situ in the NMR tube, see Figure 3a. Undecylenic acid has a terminal alkene and consequently features two resonances in the alkene region, one at a slightly lower and one at a slightly higher chemical shift compared to the internal alkene resonance of $\mathrm{OlOH}$. As a result of the stripping, sharp resonances appear in the ${ }^{1} \mathrm{H}$ NMR spectrum at around 5.5 and $4 \mathrm{ppm}$, suggesting that the molecules

associated with those resonances are no longer bound to the QDs. Indeed, the NOESY spectrum clearly confirms that these molecules are not interacting with the surface since the sharp resonances feature positive (red) NOEs (as defined by an opposite sign of the cross peak to the diagonal), indicated by the arrows, Figure 3c. The resonance at $4 \mathrm{ppm}$ is particularly interesting since this is the typical region for the $\alpha-\mathrm{CH}_{2}$ of an alcohol that has reacted to form an ester (resonance 2). In order to distinguish between the phosphonic ester of $\mathrm{OlOH}$ and TDPA or the ester of $\mathrm{OlOH}$ and undecylenic acid, ${ }^{1} \mathrm{H}-{ }^{31} \mathrm{P}$ HMQC and ${ }^{1} \mathrm{H}-{ }^{13} \mathrm{C}$ HMBC spectra were recorded. The ${ }^{1} \mathrm{H}-{ }^{31} \mathrm{P}$ HMQC (Figure 3d) revealed a coupling between resonance 2' and a phosphorous nucleus, suggesting a phosphonic ester. In contrast, correlations with carbons are absent in the ${ }^{1} \mathrm{H}-{ }^{13} \mathrm{C}$ HMBC spectrum (Figure 3e), excluding regular esters. Quantitative ${ }^{1} \mathrm{H}$ NMR analysis indicates that the total ligand density of the wurtzite CdSe QDs amounts to 3.9 ligands $/ \mathrm{nm}^{2}$, with phosphonic esters representing $25 \%$ and TDPA $75 \%$ of this total.

We thus conclude that the addition of $\mathrm{OlOH}$ eliminates (part of) the hydroxyl groups of the phosphonic acid by forming a phosphonic ester. This suppresses the tendency to form phosphonic anhydride polymers. The addition of $\mathrm{OlOH}$ also maintains the excess cadmium phosphonate in a liquid state even at room temperature, allowing their convenient separation by centrifugation. This modification in the wurtzite CdSe synthesis has made it possible to upscale the reaction to yield up to over a significant $1.5 \mu \mathrm{mol}(\approx 150 \mathrm{mg})$ of QDs produced in a single reaction. Thanks to their high purity this large amount of QDs could be stored at high concentration, dispersed in only a few $\mathrm{mL}$ of toluene.
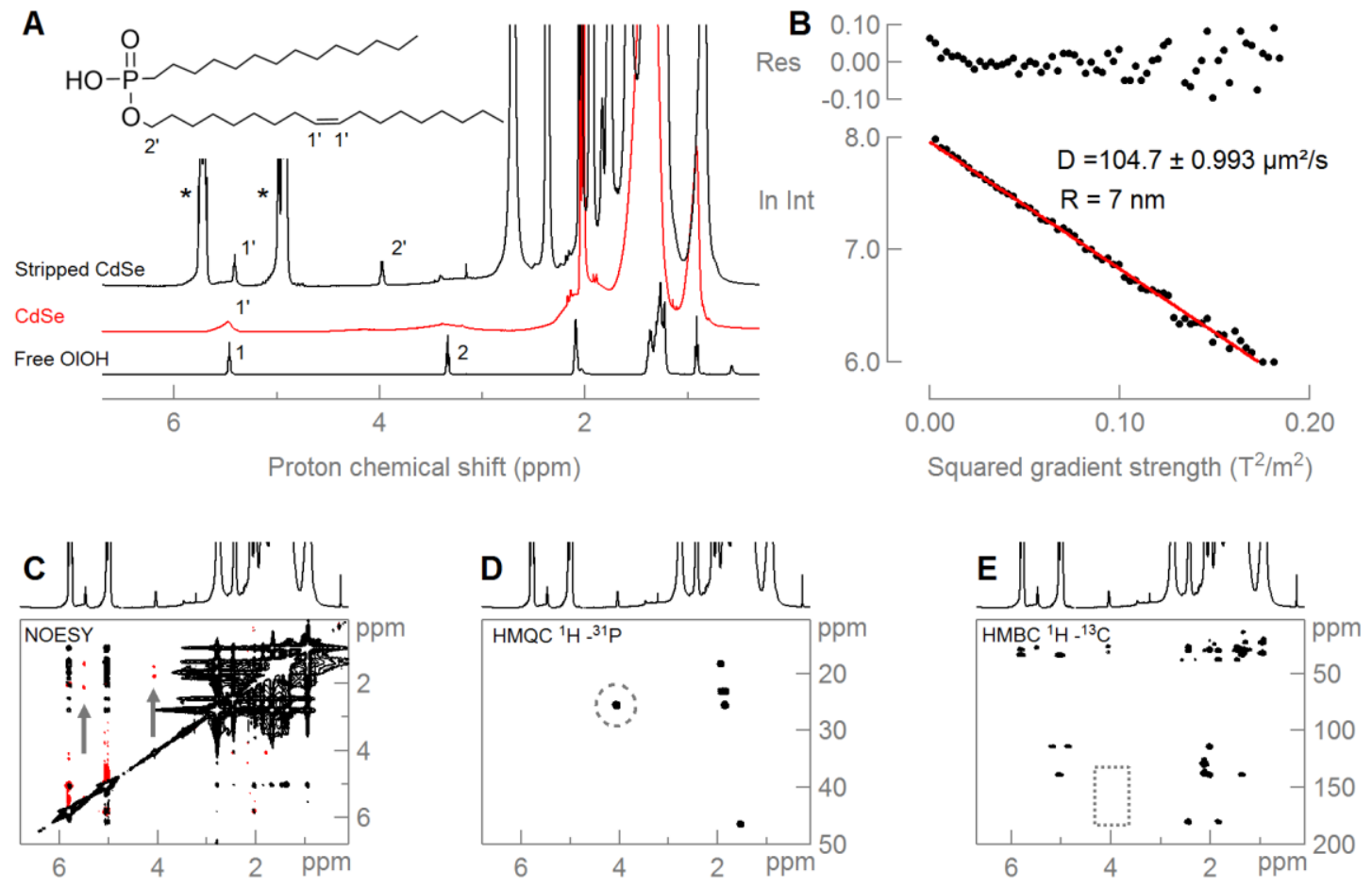

Figure 3. a) ${ }^{1} \mathrm{H}$ NMR of the free oleyl alcohol (bottom), CdSe QDs (middle) and of the in-situ stripped surface ligands of the CdSe QDs with undecylenic acid and butylamine (top) all in deuterated toluene. (1) Alkene and (2) $\mathrm{CH} 2$ resonances of the oleyl alcohol free in solution. (1') Alkene resonance and (2') the alfa $\mathrm{CH} 2$ of the alkoxy chain of phosphonic ester. (*) Alkene signals of undecylenic acid in large excess. b) DOSY mono-exponential decay of the alkene resonance on the bound phosphonic ester. c) NOESY, d) ${ }^{1} \mathrm{H}-{ }^{31} \mathrm{P}$ HMQC and e) ${ }^{1} \mathrm{H}-$ ${ }^{13} \mathrm{C}$ HMBC) spectra of the stripped phosphonic ester. The arrow points at the resonances of the exchanged phosphonic ester, while dashed circle confirms the presence of the phosphonic ester and the dashed rectangle excludes regular esters. 
The Flash Synthesis for Multi-Shell Quantum Dots. A series of modifications have been made to the flash procedure compared to the previously reported method. ${ }^{14}$ This involves the optimization and standardization of the process, geared towards the growth of different shells and an overall improvement of the quality of the resulting materials. The reaction time was extended from 3 to $5 \mathrm{~min}$. Although after $3 \mathrm{~min}$ the final size is already reached, even in the case of very thick shells, a reaction time of 5 min was found to yield somewhat more spherical QDs without affecting their size dispersion. This is often preferred in further application of these QDs but also for a more accurate concentration determination since the latter is calculated based on the volume of the QDs. A 1.2 cationic excess was used instead of an anionic excess in previous flash procedure, as this was found not to affect the quality of the QDs and to achieve optimum passivation of the outer surface. The amount of seeds was significantly upscaled from $87 \mathrm{nmol}$ to $200 \mathrm{nmol}$. Besides being an important step towards applications of these materials, larger scale syntheses at higher concentrations were also found to improve the size dispersion of the obtained QDs. A fixed amount of $2.5 \mathrm{~g}$ of oleic acid was chosen rather than a varying $\mathrm{Cd}$ :OA ratio approach. Indeed, in the case of thin shells, the amount of oleic acid added to the reaction using a ratio approach can be too small to compete with the residual phosphonic acids on the core QDs, resulting in the growth of rod-like QDs. In the case of very thick shells, the amount of injected cores can be limited to $100 \mathrm{nmol}$ so as to keep the $\mathrm{Cd}$ :OA ratio above 1:3; a value we found is the lower limit to ensure full dissolution of the $\mathrm{CdO}$ and a proper shelling. Finally, the reaction temperature was increased from 330 to $350^{\circ} \mathrm{C}$ in order to homogenize all protocols for isotropic and anisotropic shells. In addition, it was reported that syntheses of such core-shell structures at higher temperatures typically yield materials with enhanced and more robust optical properties. ${ }^{23}$
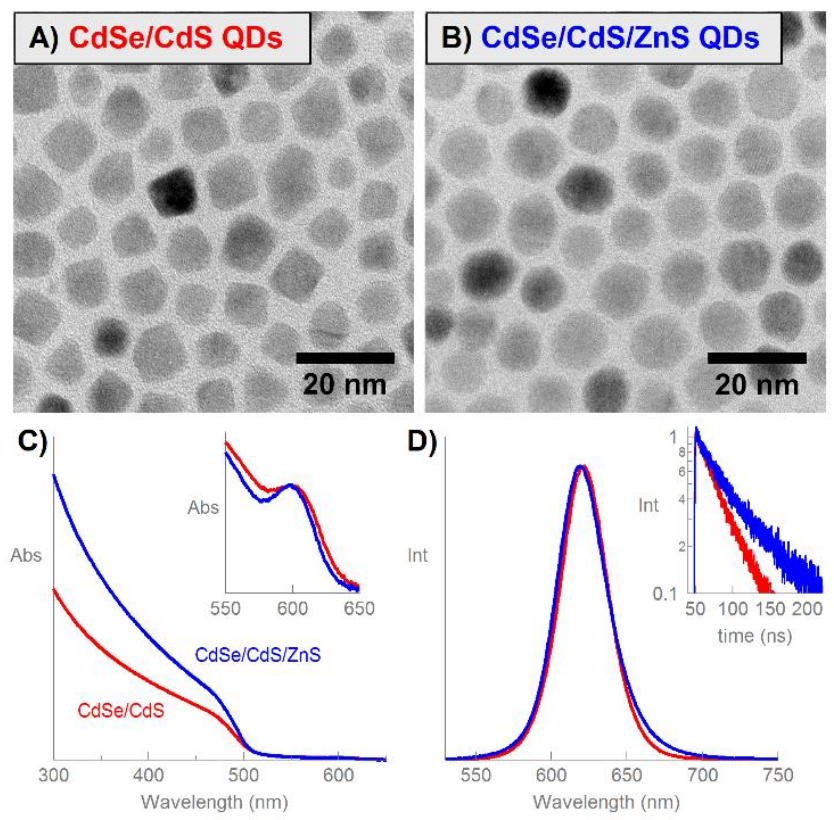

Figure 4. TEM images of a) $\mathrm{CdSe} / \mathrm{CdS}$ and b) $\mathrm{CdSe} / \mathrm{CdS} / \mathrm{ZnS}$ QDs. Optical properties with c) normalized absorption spectra (inset: focus on the excitonic feature at $600 \mathrm{~nm}$ ), d) normalized emission spectra (inset: normalized photoluminescence decays of the QDs).

CdSe/CdS QDs. Figure 4a shows an example of $9.1 \mathrm{~nm}$ $\mathrm{CdSe} / \mathrm{CdS}$ QDs obtained from this new flash procedure (see the Supporting Information, Figure S1, for more sizes). These QDs show good morphology with a size dispersion below $15 \%$, which is the case for all the QDs reported here. One should note that size dispersions below $10 \%$ can still be achieved by further increasing the concentration of the seeds in the flash synthesis (see the Supporting Information, Figure S2). Moreover, the $\mathrm{CdSe} / \mathrm{CdS}$ QDs obtained from this modified flash procedure feature improved optical properties, which are reported in Figure 4c-d for the example of the 9.1 $\mathrm{nm} \mathrm{CdSe} / \mathrm{CdS}$ QDs shown in Figure 4a. As expected for such large core-shell structures, the absorption spectrum is mostly characterized by a featureless bulk-like CdS absorption below $500 \mathrm{~nm}$, although the excitonic feature is still clearly identifiable at around $600 \mathrm{~nm}$ (inset Figure 4c). The emission spectrum is centered at $622 \mathrm{~nm}$ with a full-width half-maximum (FWHM) of only $37 \mathrm{~nm}$. Furthermore, these flash CdSe/CdS QDs show a high PLQY of $72 \%$. Another evidence of the good quality of these CdSe/CdS QDs is their PL decay, inset Figure 4d, which can be fitted well to a single exponential (resulting in a lifetime of $\tau=35.1 \mathrm{~ns}$ ). The absence of a fast component in the PL decay, which would be characteristic of non-radiative processes, is in line with the high PLQY. The enhanced quality of these flash $\mathrm{CdSe} / \mathrm{CdS}$ QDs compared to previous generation can be attributed in large part to the higher purity of the CdSe cores. Indeed, the wurtzite CdSe cores synthesized with this new synthetic protocol making use of oleyl alcohol are not only suitable for the growth of a CdS shell in spite of their slightly different surface chemistry, they also promote the growth of a much more homogeneous shell.

CdSe/CdS/ZnS QDs. Multi-shell QDs can be synthesized through a double flash method. An additional $\mathrm{ZnS}$ layer is grown on the core-shell QDs with virtually no change to the procedure except for replacing the $\mathrm{CdO}$ for $\mathrm{ZnO}$ in the reaction (see experimental section). Figure 4 shows the TEM images of corresponding $\mathrm{CdSe} / \mathrm{CdS}$ and $\mathrm{CdSe} / \mathrm{CdS} / \mathrm{ZnS}$ QDs. EDX analyses confirmed the presence of $\mathrm{Zn}$ in the $\mathrm{CdSe} / \mathrm{CdS} / \mathrm{ZnS}$ QDs (13\% relatively to the overall composition). However, in spite of aiming at a $3 \mathrm{~nm}$ thick layer in the calculations for the synthesis of the $\mathrm{ZnS}$ shell, the TEM analyses reveal that the diameter only increases from $9.1 \mathrm{~nm}$ to $10.2 \mathrm{~nm}$. This corresponds to 1 to 2 monolayers of $\mathrm{ZnS}$ and is in line with the fraction of $\mathrm{Zn}$ as measured by EDX. All attempts to increase the thickness of the $\mathrm{ZnS}$ shell by either increasing the reaction time, increasing the amount of precursors, varying the temperature and seed concentration or even trying a multi-step growth never yielded thicker $\mathrm{ZnS}$ shells. In addition the thickness of the obtained $\mathrm{ZnS}$ layer was found to be relatively independent of the size of the CdSe/CdS QDs as illustrated in the Supporting Information, Table S1. This saturation limit in growth can most likely be explained by the lattice mismatch of about $8 \%$ between the wurtzite $\mathrm{CdS}$ and wurtzite $\mathrm{ZnS}$ structures, whereas the mismatch between the wurtzite $\mathrm{CdSe}$ and wurtzite $\mathrm{CdS}$ structures is only about $4 \%$. The TEM images in Figure 4 also evidence that the QDs fully retain their size dispersion after a second flash procedure. 
Moreover the QDs appear to have an even more spherical shape.

Regarding the optical properties of these CdSe/CdS/ZnS QDs, they are compared systematically with those of the corresponding $\mathrm{CdSe} / \mathrm{CdS}$ QDs in Figure 4c-d. From point of view of absorption (Figure 4c), when normalizing the spectra at the maximum of the first exciton absorption, the growth of the $\mathrm{ZnS}$ shell translates into an increase in absorption at energies even below the bulk band-gap of $\mathrm{ZnS}(3.91 \mathrm{eV})$. It is also characterized by a more pronounced dip in the absorption spectrum after the first exciton transition (inset Figure 4c). The position of the emission peak on the other hand features a slight, 2 nm blue-shift (Figure 4d), something we consistently found for other QD sizes, except for very thin CdS layers (see the Supporting Information, Table S1). The most pronounced effect of the $\mathrm{ZnS}$ shell growth is found in the PL decay (inset Figure 4d) as it increases the lifetime from $35.1 \mathrm{~ns}$ in the case of the CdSe/CdS QDs to $53.0 \mathrm{~ns}$ for the CdSe/CdS/ZnS QDs. Although the PL decay of the CdSe/CdS/ZnS QDs seems to deviate from a pure single exponential, it is due to a long tailing rather than to a fast component that would be due to non-radiative processes. In the measurements, this long tailing is better fitted by including a constant in the fit function (see experimental section) than by adding a second exponential. This could be the sign of delayed emission due to the existence of long-lived charge-separated states as it was already reported for similar structures. ${ }^{40}$ Finally the PLQY increases from $72 \%$ to $74 \%$, which is in line with a better passivation and confinement thanks to the larger band-gap of $\mathrm{ZnS}$. Even though the increase in PLQY might seem limited, this actually confirms the good quality and passivation of the starting $\mathrm{CdSe} / \mathrm{CdS}$ QDs, which is already reflected in their high initial PLQY. Nevertheless, this increase was much more pronounced in the case of large QDs which have a lower PLQY to start with (see the Supporting Information, Table S1).

Similar changes in the optical properties of the QDs, including the blue-shift in emission as well as the increase in lifetime after the growth of a $\mathrm{ZnS}$ shell on $\mathrm{CdSe} / \mathrm{CdS}$ QDs, were already reported by Boldt et al. ${ }^{18}$ In their synthesis, the $\mathrm{ZnS}$ grows progressively owing to a rather slow continuous injection of the $\mathrm{Zn}$ and S precursors. They first observe a blue-shift at early stages of the shell growth, followed by a red-shift as the $\mathrm{ZnS}$ shell kept growing. These authors attributed the blueshift to an alloying between the $\mathrm{CdS}$ and $\mathrm{ZnS}$ shells, causing an increase in the confinement of the excitons, and the subsequent red-shift to an alloying between the CdSe core and the $\mathrm{CdS}$ due to the longer reaction time, resulting in a quasi-type II core-shell structure. They further support this claim with the increase in the lifetime after longer reaction time. However, their conclusions are somewhat contradictory with the observation made here in the case of a very fast and limited growth of a $\mathrm{ZnS}$ shell. In our case, both the blue-shift and the increase in lifetime are observed concomitantly. The blue-shift therefore cannot be explained by an increased confinement as this could only result in a better overlap of the electron and hole wave functions and in a decrease of the lifetime. This was evidenced by Diroll et al. ${ }^{41}$ who modified the method of Boldt et al. for the synthesis of $\mathrm{CdSe} / \mathrm{CdZnS}$ dot-in-rods and clearly showed the lifetime decrease with a proper alloying of $\mathrm{CdS}$ and $\mathrm{ZnS}$. Furthermore, here the blue-shift in emission is also observed for QDs with very large sizes where slight change in the confinement is not likely to have any influence on the optical properties, whereas we actually observe a red-shift for small sizes which should be much more sensitive to changes in the confinement (see the Supporting Information, Table S1). Although we cannot fully explain this discrepancy, the additional strain generated by the growth of the $\mathrm{ZnS}$ with a large lattice mismatch may affect the electronic structures of the QDs. Indeed, strain generated piezoelectric fields have been reported to becomes significantly important in such large noncentrosymmetric $\mathrm{CdSe} / \mathrm{CdS}$ core-shell structures. ${ }^{42}$

CdSe/multiCdS QDs. One of the challenges when growing QDs with very thick shells is to maintain their brightness. Indeed, while the PLQY of the QDs initially increases with the thickness of the CdS shell, it then typically decreases when reaching shells over $5 \mathrm{~nm}$ in thickness (see the Supporting Information, Table $\mathbf{S 1}$ for illustration). This is most likely due to increased strain in the structure but also due to the presence of QDs with poor morphology as highlighted in Figure 5a. The shape of those QDs sometimes even suggests that they could consist of several CdSe cores into a single structure, which would have dramatic consequence for the PLQY. Indeed, the proximity of cores within the same shell would favor energy transfer between them and increase non-radiative recombination rates. The flash synthesis offers an alternative to this limitation as multiple CdS shells can be grown on top of each other in successive flash procedures. Figure 5a shows for instance the TEM images of $14.7 \mathrm{~nm} \mathrm{CdSe/CdS} \mathrm{QDs} \mathrm{grown} \mathrm{in}$ a single step and Figure 5b shows $15.8 \mathrm{~nm} \mathrm{CdSe/CdS} \mathrm{QDs}$ where the CdS shell was grown in 3 successive steps, and therefore denoted CdSe/3.CdS. These CdSe/3.CdS QDs were grown from initially $8.5 \mathrm{~nm}$ sized $\mathrm{CdSe} / \mathrm{CdS}$ QDS, hence highlighting that in this case the growth in multi-step is not limited as in the case of $\mathrm{ZnS}$ shells. As the CdSe/3.CdS QDs show good morphology without any of the poor quality QDs visible in the large $\mathrm{CdSe} / \mathrm{CdS}$ sample, it is also characterized by a PLQY of $64 \%$ which is considerably better than the $23 \%$ of the $\mathrm{CdSe} / \mathrm{CdS}$ QDs in Figure 5a. Furthermore, these CdSe/3.CdS QDs show a 140-fold increase between their absorption coefficient at the first excitonic peak and at 450 $\mathrm{nm}$. A property which is of high importance to prevent selfabsorption when using QDs in solar concentrators for instance. $^{43,44}$

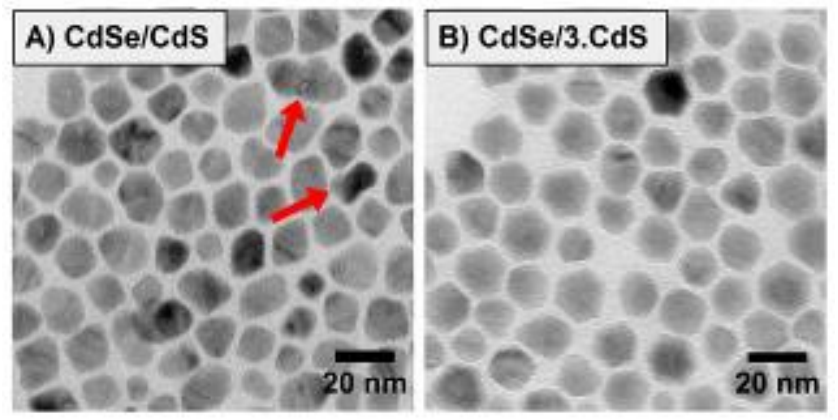

Figure 5. TEM images of a) $\mathrm{CdSe} / \mathrm{CdS}$ QDs grown in a single step, and b) CdSe/3.CdS QDs grown in multiple steps. Red arrows in a) to highlight QDs with poor morphology. 

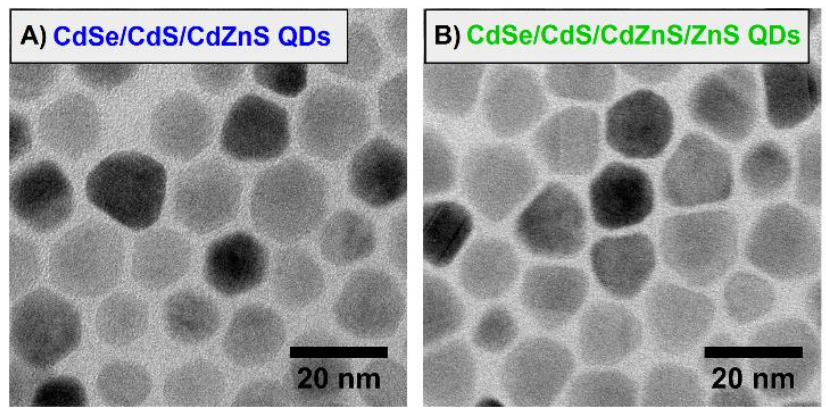

c)
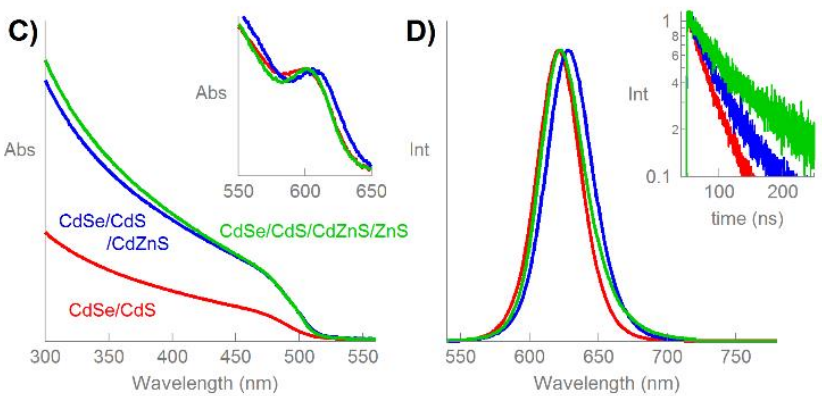

Figure 6. TEM images of a) $\mathrm{CdSe} / \mathrm{CdS} / \mathrm{CdZnS}$ and b) $\mathrm{CdSe} / \mathrm{CdS} / \mathrm{CdZnS} / \mathrm{ZnS}$ QDs. Optical properties with c) normalized absorption spectra (inset: focus on the excitonic feature at $600 \mathrm{~nm}$ ), d) normalized emission spectra (inset: normalized photoluminescence decays of the QDs).

Alloyed CdZnS shells. The high versatility of the flash method is further demonstrated through the growth of alloyed $\mathrm{CdZnS}$ shells. As described in the experimental part, this is simply achieved by mixing $\mathrm{CdO}$ and $\mathrm{ZnO}$ in the reaction flask prior to injection of the seed QDs and sulfur precursor. All the parameters for the growth of such alloyed shells remain identical as for the growth of a $\mathrm{CdS}$ or $\mathrm{ZnS}$ shell. Figure 6a shows the TEM image of $\mathrm{CdSe} / \mathrm{CdS} / \mathrm{CdZnS}$ QDs of $12.3 \mathrm{~nm}$ in size grown from the same $9.1 \mathrm{~nm} \mathrm{CdSe/CdS} \mathrm{QDs} \mathrm{as} \mathrm{shown} \mathrm{in}$ Figure 4a. It corresponds to the growth of a $1.6 \mathrm{~nm}$ thick shell, in spite of aiming at a $3 \mathrm{~nm}$ thick shell in the synthesis calculations. In addition, the synthesis was made with a molar ratio of $75 \% \mathrm{Zn}$ for $25 \% \mathrm{Cd}$, whereas EDX analyses revealed that the additional shell only contains $15 \% \mathrm{Zn}$ (relatively to the cations composition). This lower amount of $\mathrm{Zn}$ compared to the theoretical ratio in the reaction mixture is line with the growth of a thinner shell and can be reasonably explained by either a lower reactivity of the $\mathrm{Zn}$ precursor and the limited growth of a pure $\mathrm{ZnS}$ shell even after all the $\mathrm{Cd}$ has reacted for the same reason as for $\mathrm{CdSe} / \mathrm{CdS} / \mathrm{ZnS}$ QDs. Thus, although this was not investigated in details, it is more likely that the $\mathrm{CdZnS}$ shell is not made of a homogeneous alloy of $\mathrm{Cd}$ and $\mathrm{Zn}$, but rather features a gradient composition with increasing $\mathrm{Zn}$ content towards the QD surface. Indeed, when attempting to further grow an additional $3 \mathrm{~nm}$ thick $\mathrm{ZnS}$ shell on top of these $\mathrm{CdSe} / \mathrm{CdS} / \mathrm{CdZnS}$ QDs, the QDs did not grow significantly in size. Figure $\mathbf{6 b}$ shows the TEM image of $\mathrm{CdSe} / \mathrm{CdS} / \mathrm{CdZnS} / \mathrm{ZnS}$ QDs obtained with a triple flash procedure, which were also measured to have an average diameter of $12.3 \mathrm{~nm}$. Nevertheless, EDX analyses showed an increase of the $\mathrm{Zn}$ content, from $15 \%$ in the $\mathrm{CdZnS}$ shell to $35 \%$ in the $\mathrm{CdZnS} / \mathrm{ZnS}$ shell. This increase in the $\mathrm{Zn}$ content was not accompanied by a decrease of the $\mathrm{Cd}$ content, as determined by comparing to the amount of $\mathrm{Se}$ which is considered to remain constant throughout the shell additions. This rules out a possible cationic exchange and confirms the effective growth of an additional $\mathrm{ZnS}$ layer. According to the EDX analyses, the additional $\mathrm{ZnS}$ layer should translate in an volume increase of about $10 \%$. This increase in volume is however not reflected by an increase in diameter measurable by TEM analysis due to a reshaping of the QDs towards a more spherical shape.

Regarding their optical properties, these QDs with alloyed $\mathrm{CdZnS}$ layers are compared together with the starting $\mathrm{CdSe} / \mathrm{CdS}$ QDs in Figure 6c-d. The growth of a CdZnS layer results in a more than 2-fold increase of the absorbance below $500 \mathrm{~nm}$. It also induces a red-shift of both the excitonic absorption and emission peaks. This increase in absorbance and red-shifts are easily explained by the large amount of $\mathrm{Cd}$ in the additional layer, as they both are consequences of a larger $\mathrm{CdS}$ volume for the same amount of $\mathrm{CdSe}$, allowing the electron to further delocalize in the shell. This also explains the increase in lifetime from $35.1 \mathrm{~ns}$ to $45.5 \mathrm{~ns}$ (inset Figure 6d), due to a lower overlap between the electron and hole wave functions. The growth of an additional $\mathrm{ZnS}$ shell on these $\mathrm{CdSe} / \mathrm{CdS} / \mathrm{CdZnS}$ QDs causes again a faint, yet significant, increase in absorption at higher energies and a more pronounced dip on the blue side of the excitonic absorption peak (Figure 6c). It also induces a relatively large blue-shift of the emission peak (Figure 6d) and a concomitant increase of the lifetime, from 45.5 to $76.1 \mathrm{~ns}$ (inset Figure 6d). All these observations are in line with those made in the case of $\mathrm{CdSe} / \mathrm{CdS} / \mathrm{ZnS}$ QDs, which confirms the effective growth of the $\mathrm{ZnS}$ shell in spite of the limited size increase seen by TEM analyses. Nevertheless, the $7 \mathrm{~nm}$ blue-shift of the emission spectrum observed in $\mathrm{CdSe} / \mathrm{CdS} / \mathrm{CdZnS} / \mathrm{ZnS}$ QDs (Figure 6d) is much more pronounced than in the case of non-alloyed $\mathrm{CdSe} / \mathrm{CdS} / \mathrm{ZnS}$ QDs where the blue-shift was limited to $2 \mathrm{~nm}$ (Figure 4d). This is truly due to the nature of the alloyed $\mathrm{CdZnS}$ layer, as the same observation is made for $\mathrm{CdSe} / \mathrm{CdZnS} / \mathrm{ZnS}$ QDs, i.e. when an alloyed $\mathrm{CdZnS}$ shell is grown directly on the $\mathrm{CdSe}$ cores without any intermediate CdS layer (see the Supporting Information, Figure S3). Here again, the blue-shift cannot be attributed to a larger alloying of $\mathrm{Zn}$ in the $\mathrm{CdZnS}$ layer, because an increased exciton confinement should also translate in a decrease of the lifetime.

Nevertheless, the alloyed layer also benefits the PL efficiency of these QDs. Indeed, the successive shelling with a $\mathrm{CdZnS}$ alloy and pure $\mathrm{ZnS}$ increases the PLQY from $72 \%$ for the starting CdSe/CdS QDs to 73\% and then 76\%, respectively. Thus, although the increase in PLQY is limited, it is still higher than for the CdSe/CdS/ZnS QDs (74\%), and this in spite of the larger QD size, which indicates enhanced surface passivation with minimal additional strain induced, probably thanks to the alloyed layer.

The Flash Synthesis for Multi-Shell Nanorods. As mentioned in the introduction, the flash synthesis actually originates from the dot-in-rod synthesis reported by Carbone et $a l .{ }^{19}$ These anisotropic structures are obtained by using a mixture of a short and a long phosphonic acids for the dissolution of $\mathrm{CdO}$ and stabilization of the NRs. This section will now show that the flash procedure can also be used for the growth of a $\mathrm{ZnS}$ on these dot-in-rod structures just like in the case of spherical QDs, hence highlighting once again the great versatility of the flash technique. However, we have also previously reported that $\mathrm{CdSe} / \mathrm{CdS}$ NRs synthesized with the protocol of Carbone et al. yields NRs which feature physical connections. This was notably evidenced through the silica encapsulation of 
these NRs (Figure 7a), resulting in many nanoparticles containing two NRs. When primarily attempting to grow a $\mathrm{ZnS}$ shell on such CdSe/CdS NRs, the connections remained (Figure 7b), which is undesirable for optimal optical properties and application of these NRs. Although the nature and origin of these connection were initially not understood, TEM observations evidenced that some NRs are connected through their sharp tip ends (Figure 7c). Since similar ends of these NRs with a non-centrosymmetric wurtzite structure should have the same polarity, this observation rules out the hypothesis of dipolar interactions being at the origin of the connections.
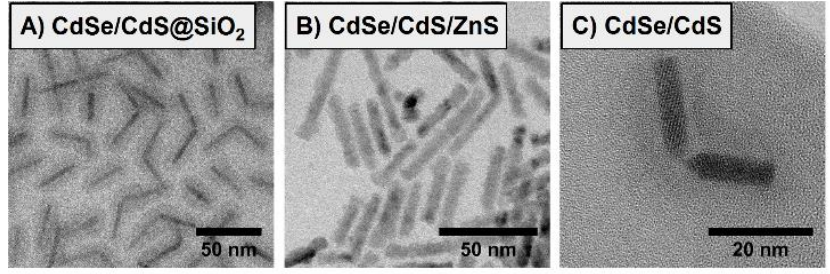

Figure 7. TEM images of a) silica coated $\mathrm{CdSe} / \mathrm{CdS} \mathrm{NRs}$, b) $\mathrm{CdSe} / \mathrm{CdS} / \mathrm{ZnS}$ NRs grown from connected CdSe/CdS NRs, and c) two CdSe/CdS NRs connected through their tips.
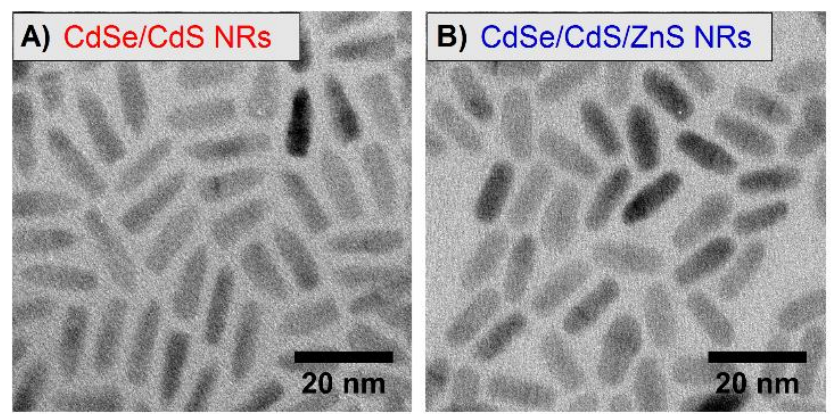

C)

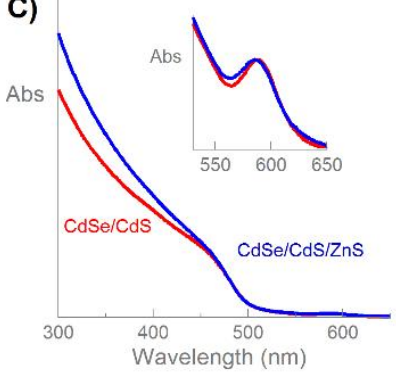

D)

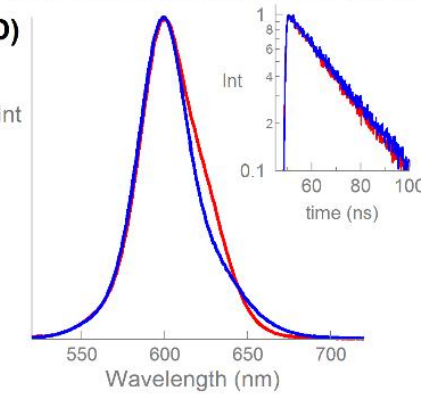

Figure 8. TEM images of a) $\mathrm{CdSe} / \mathrm{CdS}$ and b) $\mathrm{CdSe} / \mathrm{CdS} / \mathrm{ZnS}$ NRs. Optical properties with c) normalized absorption spectra (inset: focus on the excitonic feature at $590 \mathrm{~nm}$ ), d) normalized emission spectra (inset: normalized photoluminescence decays of the NRs).

CdSe/CdS NRs. The original CdSe/CdS NRs as reported by Carbone et al. ${ }^{19}$ were synthesized with a mixture of hexylphosphonic acid (HPA) as the short acid and octadecylphosphonic acid (ODPA) as the long acid. In this work, the short acid was changed for dodecylphosphonic acid (DDPA) and most parameters were adjusted for better standardization with the flash procedure. Only few parameters differ from the synthesis of spherical $\mathrm{CdSe} / \mathrm{CdS}$ QDs. The reaction time for the synthesis of NRs was extended to $8 \mathrm{~min}$ as TEM observations have shown substantial growth of the NRs even after 5 min of reaction. The total amount of phosphonic acid was set to the minimal molar ratio of 3:1 compared to the $\mathrm{CdO}$, mostly due to the high cost of these chemicals. The molar ratio ODPA:DDPA was also kept at 1.5 , because decreasing this ratio typically caused a destabilization of the NRs during the reaction, indicating that a high amount of the long acid is necessary to preserve the colloidal stability of these relatively large objects, even in spite of increasing the length of the short acid. As for the synthesis of the core CdSe QDs, the use of phosphonic acids for the NRs synthesis can also result in the formation of a phosphonic anhydride polymer and cause gelification of the NRs suspensions. Unfortunately, in this case the use of oleyl alcohol to prevent this was unsuccessful due to formation of an insoluble complex, even at high temperature, with the Cd and ODPA. Thus, to prevent extensive precipitation of a phosphonic acid polymer, the first purification step of the NRs was done while the reaction mixture was still warm as detailed in the experimental section. However, this technique is only efficient for small amount of phosphonic acid in the reaction and the amount of $\mathrm{CdSe}$ seeds was therefore limited to $50 \mathrm{nmol}$ for the synthesis of the CdSe/CdS NRs.

Figure 8 shows the TEM image of $5.1 \times 15.3 \mathrm{~nm} \mathrm{CdSe} / \mathrm{CdS}$ NRs obtained from the modified synthesis mentioned above, evidencing that the use of DDPA instead of HPA still promotes the growth of an anisotropic shell. More importantly, these NRs are free of connections, as demonstrated through their subsequent silica encapsulation (see the Supporting Information, Figure $\mathbf{S 4}$ for TEM image and experimental details). In the light of this result, it can be reasonably assumed that the tips of the NRs are predominantly passivated by the short chain acid, offering less steric hindrance at these locations and therefore enabling NRs to make direct contact. Once in this situation, the Van der Waals interactions are too strong to allow dissociation of the NRs. Here, the substitution of HPA (6 carbons chain) for DDPA (12 carbons chain) offers better steric passivation to prevent the connections between NRs. Intermediate phosphonic acids with increasing carbon chain lengths have also been tested. The amount of connections between rods decreased with increasing the length of the chain, but only at a carbon chain of 12 did the connections between NRs completely cease to occur.

CdSe/CdS/ZnS NRs. The exact same ZnS shelling procedure as for the spherical QDs was applied to the CdSe/CdS NRs. Figure 8 shows the TEM images of corresponding CdSe/CdS and $\mathrm{CdSe} / \mathrm{CdS} / \mathrm{ZnS}$ NRs. After this second flash step, the NRs grow from $5.1 \times 15.3 \mathrm{~nm}$ to $5.9 \times 15.8 \mathrm{~nm}$, which corresponds to a single monolayer of $\mathrm{ZnS}$. The growth of the $\mathrm{ZnS}$ shell was confirmed by EDX analyses which indicated a $\mathrm{Zn}$ content of $14 \%$ relatively to the overall composition of the $\mathrm{CdSe} / \mathrm{CdS} / \mathrm{ZnS}$ NRs. This indicates that additional shells can effectively be grown through a flash procedure even on these anisotropic structures which are initially passivated with tightly bound phosphonic acids. The seemingly limited growth in the long direction is more likely due to the rounding of the sharp end facets of the NR tips, rather than to an uncomplete $\mathrm{ZnS}$ layer. In spite of this rounding, which is an expected consequence of the system reducing the free energy, the NRs virtually retain their aspect ratio, which only decreases from 3 to 2.7. Moreover, Figure $\mathbf{8 b}$ also evidences that these $\mathrm{CdSe} / \mathrm{CdS} / \mathrm{ZnS}$ NRs, which are now passivated with oleic acid instead of phosphonic acids, are free of connections. This is in clear contrast with those in Figure $\mathbf{7 b}$, which highlights the importance of the modification in the CdSe/CdS NR synthesis. This is further demonstrated through the silica encapsulation 
of the $\mathrm{CdSe} / \mathrm{CdS} / \mathrm{ZnS}$ NRs (see the Supporting Information, Figure S4).

Regarding their optical properties (Figure 8c and d), the growth of the $\mathrm{ZnS}$ shell again increases the absorbance at high energies (Figure 8c). However, in contrast with the spherical QDs it does not cause a more pronounced dip after the excitonic feature in the absorption spectrum, nor significantly shifts the emission peak. Even more obvious, the growth of the $\mathrm{ZnS}$ does not affect the PL decay of these NRs (inset Figure 8d), with measured lifetimes of $20.2 \mathrm{~ns}$ and $21.5 \mathrm{~ns}$ for the $\mathrm{CdSe} / \mathrm{CdS}$ and $\mathrm{CdSe} / \mathrm{CdS} / \mathrm{ZnS}$ NRs respectively. On the one hand the lifetime doesn't decrease, indicating that there is virtually no or very limited alloying between the CdS and $\mathrm{ZnS}$ shells as this would have increased the overlap between the hole and electron wave functions as previously reported ${ }^{41}$ and discussed above. On the other hand, neither did the lifetime increase, as it was the case for spherical QDs. This is also in line with the absence of a strain induced piezoelectric field due to the relatively short diameter of these NRs, even in spite of their anisotropic shape and their long dimension being beyond the Bohr radius of the material. Figure 8d shows that the shape of the emission spectrum is affected by the growth of the $\mathrm{ZnS}$ shell. The $\mathrm{CdSe} / \mathrm{CdS}$ NRs feature a small shoulder on the red side of their emission spectrum probably due to NRs with lower aspect ratio and larger diameter as visible in Figure 8a. In the $\mathrm{CdSe} / \mathrm{CdS} / \mathrm{ZnS}$ NRs this shoulder is smoothed out towards a more symmetrical spectrum. Although this could be explained by the PLQY of different NRs being affected to different extent by the $\mathrm{ZnS}$ shell, this effect is however minor as both samples showed an equal PLQY of $80 \%$.

\section{CONCLUSION}

We have developed a generic flash method for the formation of a variety of Cd-based core/multiple shell QDs and NRs. The method builds on an improved synthesis of wurtzite CdSe core QDs, where we introduce oleyl alcohol to prevent the polymerization of the phosphonic acid through the formation of a phosphonic ester. This simple change in the reaction allowed for the production of large amounts of high purity core QDs which is key for the subsequent synthesis of high quality core-shell structures. The versatility of the flash method is demonstrated through the synthesis of multi-shell QDs and NRs. We have shown that a simple unique procedure can be applied multiple times for the successive growth of not only $\mathrm{CdS}$ and $\mathrm{ZnS}$ but also $\mathrm{CdZnS}$ alloyed shells. This synthesis strategy has proven its efficiency to overcome several limitations for the growth of shells in spite of the lattice mismatch and different surface chemistry. This resulted in materials with enhanced optical properties, even in the case of very thick $\mathrm{CdS}$ shells. The high versatility of this technique opens up numerous opportunities for the synthesis of heterostructures covering a wide range of materials beyond those reported here.

\section{ASSOCIATED CONTENT}

Supporting information. Characterization of additional samples including TEM images, table with optical properties, absorption and photoluminescent spectra, PL decays. This material is available free of charge via the Internet at http://pubs.acs.org.

\section{AUTHOR INFORMATION}

\section{Corresponding Authors}

*E-mail: Zeger.Hens@ugent.be
*E-mail: Tangi.Aubert@ugent.be

\section{Notes}

The authors declare no competing financial interest.

\section{ACKNOWLEDGMENT}

The authors acknowledge European Comission via the MarieSklodowska Curie action Phonsi (H2020-MSCA-ITN-642656), BelSPo (IAP 7.35, photonics@be), FWO-Vlaanderen (KaN 1509012N), Ghent University (BOF12/GOA/ (GOA 01G01513; $\mathrm{BOF} 14 / \mathrm{PDO} / 007)$ and IWT-Vlaanderen for financial support.

\section{REFERENCES}

1. Jaiswal, J. K.; Simon, S. M., Potentials and Pitfalls of Fluorescent Quantum Dots for Biological Imaging. Trends Cell. Biol. 2004, 14, (9), 497-504.

2. Chan, W. C. W.; Maxwell, D. J.; Gao, X.; Bailey, R. E.; Han, M.; Nie, S., Luminescent Quantum Dots for Multiplexed Biological Detection and Imaging. Curr. Opin. Biotechnol. 2002, 13, (1), 40-46.

3. McDonald, S. A.; Konstantatos, G.; Zhang, S.; Cyr, P. W.; Klem, E. J.; Levina, L.; Sargent, E. H., Solution-Processed PbS Quantum Dot Infrared Photodetectors and Photovoltaics. Nat. Mater. 2005, 4, (2), 138-142.

4. Lee, S. F.; Osborne, M. A., Brightening, Blinking, Bluing and Bleaching in the Life of a Quantum Dot: Friend or Foe? Chem. Phys. Chem. 2009, 10, (13), 2174-2191.

5. Frantsuzov, P.; Kuno, M.; Jankó, B.; Marcus, R. A., Universal Emission Intermittency in Quantum Dots, Nanorods and Nanowires. Nat. Phys. 2008, 4, (5), 519-522.

6. Heyes, C. D.; Kobitski, A. Y.; Breus, V. V.; Nienhaus, G. U., Effect of the Shell on the Blinking Statistics of Core-Shell Quantum Dots: A Single-Particle Fluorescence Study. Phys. Rev. B: Condens. Matter 2007, 75, (12), 125431125438 .

7. de Mello Donega, C., Synthesis and Properties of Colloidal Heteronanocrystals. Chem. Soc. Rev. 2011, 40, (3), 1512-1546.

8. Eychmüller, A., Structure and Photophysics of Semiconductor Nanocrystals. $J$. Phys. Chem. B 2000, 104, (28), 6514-6528.

9. Chen, Y.; Vela, J.; Htoon, H.; Casson, J. L.; Werder, D. J.; Bussian, D. a.; Klimov, V. I.; Hollingsworth, J. a., "Giant" Multishell CdSe Nanocrystal Quantum Dots with Suppressed 
Blinking. J. Am. Chem. Soc. 2008, 130, 50265027.

10. Mahler, B.; Spinicelli, P.; Buil, S.; Quelin, X.; Hermier, J.-P.; Dubertret, B., Towards Non-Blinking Colloidal Quantum Dots. Nat. Mater. 2008, 7, 659-664.

11. Chen, O.; Zhao, J.; Chauhan, V. P.; Cui, J.; Wong, C.; Harris, D. K.; Wei, H.; Han, H. S.; Fukumura, D.; Jain, R. K.; Bawendi, M. G., Compact High-Quality CdSe-CdS Core-Shell Nanocrystals with Narrow Emission Linewidths and Suppressed Blinking. Nat. Mater. 2013, 12, (5), 445-451.

12. Christodoulou, S.; Vaccaro, G.; Pinchetti, V.; De Donato, F.; Grim, J. Q.; Casu, A.; Genovese, A.; Vicidomini, G.; Diaspro, A.; Brovelli, S.; Manna, L.; Moreels, I., Synthesis of Highly Luminescent Wurtzite CdSe/CdS GiantShell Nanocrystals Using a Fast Continuous Injection Route. J. Mater. Chem. C 2014, 2, (17), 3439-3447.

13. Nasilowski, M.; Spinicelli, P.; Patriarche, G.; Dubertret, B., Gradient CdSe/CdS Quantum Dots with Room Temperature Biexciton Unity Quantum Yield. Nano Lett. 2015, 15, (6), 39533958.

14. Cirillo, M.; Aubert, T.; Gomes, R.; Deun, R. V.; Emplit, P.; Biermann, A.; Lange, H.; Thomsen, C.; Brainis, E.; Hens, Z., "Flash" Synthesis of $\mathrm{CdSe} / \mathrm{CdS}$ Core-Shell Quantum Dots. Chem. Mater. 2014, 26, 1154-1160.

15. Aubert, T.; Soenen, S. J.; Wassmuth, D.; Cirillo, M.; Deun, R. V.; Braeckmans, K.; Hens, Z., Bright and Stable CdSe/CdS@SiO2 Nanoparticles Suitable for Long-Term Cell Labeling. ACS Appl. Mater. Interfaces 2014, 6, 11714-11723.

16. Xie, W.; Zhu, Y.; Aubert, T.; Hens, Z.; Brainis, E.; Van Thourhout, D., Fabrication and Characterization of On-Chip Silicon Nitride Microdisk Integrated with Colloidal Quantum Dots. Opt. Express 2016, 24, (2), A114-A122.

17. Xie, W.; Zhu, Y.; Aubert, T.; Verstuyft, S.; Hens, Z.; Van Thourhout, D., Low-Loss Silicon Nitride Waveguide Hybridly Integrated with Colloidal Quantum Dots. Opt. Express 2015, 23, (9), 12152-12160.

18. Boldt, K.; Kirkwood, N.; Beane, G. A.; Mulvaney, P., Synthesis of Highly Luminescent and Photo-Stable, Graded Shell CdSe/CdxZn1-
xS Nanoparticles by In Situ Alloying. Chem. Mater. 2013, 25, (23), 4731-4738.

19. Carbone, L.; Nobile, C.; De Giorgi, M.; Sala, F. D.; Morello, G.; Pompa, P.; Hytch, M.; Snoeck, E.; Fiore, A.; Franchini, I. R.; Nadasan, M.; Silvestre, A. F.; Chiodo, L.; Kudera, S.; Cingolani, R.; Krahne, R.; Manna, L., Synthesis and Micrometer-Scale Assembly of Colloidal $\mathrm{CdSe} / \mathrm{CdS}$ Nanorods Prepared by a Seeded Growth Approach. Nano lett. 2007, 7, 29422950.

20. Hu, J.; Li, L.; Yang, W.; Manna, L.; Wang, L.; Alivisatos, A. P., Linearly Polarized Emission from Colloidal Semiconductor Quantum Rods. Science 2001, 292, (5524), 20602063.

21. Ahmed, S.; Ryan, K. M., Centimetre Scale Assembly of Vertically Aligned and Close Packed Semiconductor Nanorods from Solution. Chem. Commun. 2009, (42), 6421-6423.

22. Aubert, T.; Palangetic, L.; Mohammadimasoudi, M.; Neyts, K.; Beeckman, J.; Clasen, C.; Hens, Z., Large-Scale and Electroswitchable Polarized Emission from Semiconductor Nanorods Aligned in Polymeric Nanofibers. ACS Photonics 2015, 2, (5), 583-588. 23. Diroll, B. T.; Murray, C. B., HighTemperature Photoluminescence of $\mathrm{CdSe} / \mathrm{CdS}$ Core/Shell Nanoheterostructures. ACS Nano 2014, 8, (6), 6466-6474.

24. Cao, G.; Rabenberg, L. K.; Nunn, C. M.; Mallouk, T. E., Formation of Quantum-Size Semiconductor Particles in a Layered Metal Phosphonate Host Lattice. Chem. Mater. 1991, 3, (1), 149-156.

25. Cao, G.; Lynch, V. M.; Yacullo, L. N., Synthesis, Structural Characterization, and Intercalation Chemistry of Two Layered Cadmium Organophosphonates. Chem. Mater. 1993, 5, (7), 1000-1006.

26. Liu, H.; Owen, J. S.; Alivisatos, A. P., Mechanistic Study of Precursor Evolution in Colloidal Group II-VI Semiconductor Nanocrystal Synthesis. J. Am. Chem. Soc. 2007, 129, (2), 305-312.

27. Owen, J. S.; Chan, E. M.; Liu, H.; Alivisatos, A. P., Precursor Conversion Kinetics and the Nucleation of Cadmium Selenide Nanocrystals. J. Am. Chem. Soc. 2010, 132, (51), 18206-18213. 
28. Jasieniak, J.; Smith, L.; Embden, J. v.; Mulvaney, P.; Califano, M., Re-Examination of the Size-Dependent Absorption Properties of CdSe Quantum Dots. J. Phys. Chem. C 2009, 113, (45), 19468-19474.

29. De Geyter, B.; Hens, Z., The Absorption Coefficient of $\mathrm{PbSe} / \mathrm{CdSe}$ Core/Shell Colloidal Quantum Dots. Appl. Phys. Lett. 2010, 97, (16), 161908-161910.

30. Hens, Z.; Moreels, I., Light Absorption by Colloidal Semiconductor Quantum Dots. J. Mater. Chem. 2012, 22, (21), 10406-10415.

31. de Mello, J. C.; Wittmann, H. F.; Friend, R. H., An Improved Experimental Determination of External Photoluminescence Quantum Efficiency. Adv. Mater. 1997, 9, (3), 230-232.

32. Connell, M. A.; Bowyer, P. J.; Adam Bone, P.; Davis, A. L.; Swanson, A. G.; Nilsson, M.; Morris, G. A., Improving the Accuracy of Pulsed Field Gradient NMR Diffusion Experiments: Correction for Gradient NonUniformity. J. Magn. Reson. 2009, 198, (1), 121131.

33. Sinnaeve, D., The Stejskal-Tanner Equation Generalized for Any Gradient ShapeAn Overview of Most Pulse Sequences Measuring Free Diffusion. Concepts Magn. Reson. 2012, 40A, (2), 39-65.

34. Hens, Z.; Martins, J. C., A Solution NMR Toolbox for Characterizing the Surface Chemistry of Colloidal Nanocrystals. Chem. of Mater. 2013, 25, 1211-1221.

35. De Roo, J.; Van Driessche, I.; Martins, J. C.; Hens, Z., Colloidal Metal Oxide Nanocrystal Catalysis by Sustained Chemically Driven Ligand Displacement. Nat. Mater. 2016, 15, (5), 517-21.

36. Gomes, R.; Hassinen, A.; Szczygiel, A.; Zhao, Q.; Vantomme, A.; Martins, J. C.; Hens, Z., Binding of Phosphonic Acids to CdSe Quantum Dots: A Solution NMR Study. J. Phys. Chem. Lett. 2011, 2, (3), 145-152.

37. De Roo, J.; Van den Broeck, F.; De Keukeleere, K.; Martins, J. C.; Van Driessche, I.; Hens, Z., Unravelling the Surface Chemistry of Metal Oxide Nanocrystals, the Role of Acids and Bases. J. Am. Chem. Soc. 2014, 136, (27), 96509657.
38. Kopping, J. T.; Patten, T. E., Identification of Acidic Phosphorus-Containing Ligands Involved in the Surface Chemistry of CdSe Nanoparticles Prepared in Tri-NOctylphosphine Oxide Solvents. J. Am. Chem. Soc. 2008, 130, (17), 5689-5698.

39. Morris-Cohen, A. J.; Donakowski, M. D.; Knowles, K. E.; Weiss, E. A., The Effect of a Common Purification Procedure on the Chemical Composition of the Surfaces of CdSe Quantum Dots Synthesized with Trioctylphosphine Oxide. J. Phys. Chem. C 2010, 114, (2), 897-906.

40. Rabouw, F. T.; Kamp, M.; van DijkMoes, R. J.; Gamelin, D. R.; Koenderink, A. F.; Meijerink, A.; Vanmaekelbergh, D., Delayed Exciton Emission and Its Relation to Blinking in CdSe Quantum Dots. Nano Lett. 2015, 15, (11), 7718-7725.

41. Diroll, B. T.; Turk, M. E.; Gogotsi, N.; Murray, C. B.; Kikkawa, J. M., Ultrafast Photoluminescence from the Core and the Shell in $\mathrm{CdSe} / \mathrm{CdS}$ Dot-in-Rod Heterostructures. Chem. Phys. Chem. 2016, 17, (5), 759-765.

42. Christodoulou, S.; Rajadell, F.; Casu, A.; Vaccaro, G.; Grim, J. Q.; Genovese, A.; Manna, L.; Climente, J. I.; Meinardi, F.; Raino, G.; Stoferle, T.; Mahrt, R. F.; Planelles, J.; Brovelli, S.; Moreels, I., Band Structure Engineering via Piezoelectric Fields in Strained Anisotropic CdSe/CdS Nanocrystals. Nat. Commun. 2015, 6, 7905-7913.

43. Meinardi, F.; Colombo, A.; Velizhanin, K. A.; Simonutti, R.; Lorenzon, M.; Beverina, L.; Viswanatha, R.; Klimov, V. I.; Brovelli, S., Large-Area Luminescent Solar Concentrators Based on 'Stokes-Shift-Engineered' Nanocrystals in a Mass-Polymerized PMMA Matrix. Nat. Photonics 2014, 8, (5), 392-399.

44. Bronstein, N. D.; Li, L.; Xu, L.; Yao, Y.; Ferry, V. E.; Alivisatos, A. P.; Nuzzo, R. G., Luminescent Solar Concentration with Semiconductor Nanorods and Transfer-Printed Micro-Silicon Solar Cells. ACS Nano 2014, 8, (1), 44-53. 



\title{
Revisited Wurtzite CdSe Synthesis : a Gateway for the Versatile Flash Synthesis of Multi-Shell Quantum Dots and Rods
}

\author{
Emile Drijvers ${ }^{1,3}$, Jonathan De Roo ${ }^{2}$, Krisztina Fehér ${ }^{4}$, Zeger Hens ${ }^{1,3}$ and Tangi Aubert ${ }^{1,3}$ \\ ${ }^{1}$ Physics and Chemistry of Nanostructures (PCN), and ${ }^{2}$ Sol-gel Centre for Research on Inorganic Powders and Thin films Synthesis \\ (SCRiPTS), Department of Inorganic and Physical Chemistry, Ghent University, Krijgslaan 281-S3, 9000 Ghent, Belgium. \\ ${ }^{3}$ Center for Nano and Biophotonics (NB-Photonics), Ghent University, Sint-Pietersnieuwstraat 41, 9000 Ghent, Belgium. \\ ${ }^{4}$ NMR and Structure Analysis Unit, Department of Organic and Macromolecular Chemistry, Ghent University, Krijgslaan 281-S4, 9000 \\ Ghent, Belgium.
}

KEYWORDS: Semiconductor, nanocrystal, NMR, CdSe/CdS/ZnS, giant core-shell.

Corresponding authors: Zeger.Hens@UGent.be, Tangi.Aubert@UGent.be

Content: Additional TEM images, table of optical properties, absorption and photoluminescent spectra, photoluminescent decays. 


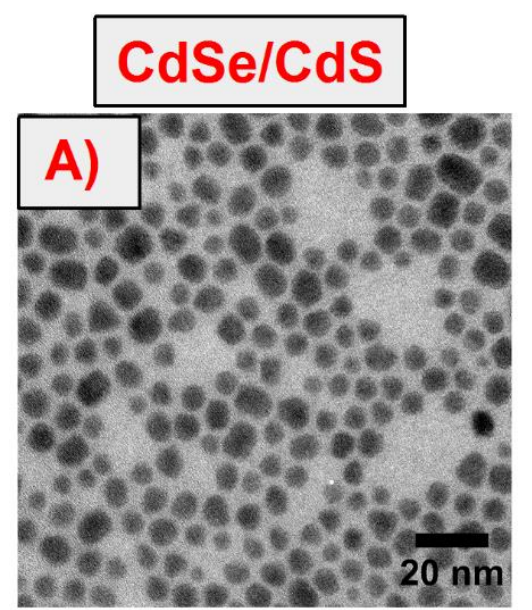

\section{$\mathrm{CdSe} / \mathrm{CdS} / \mathrm{ZnS}$}

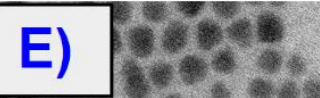
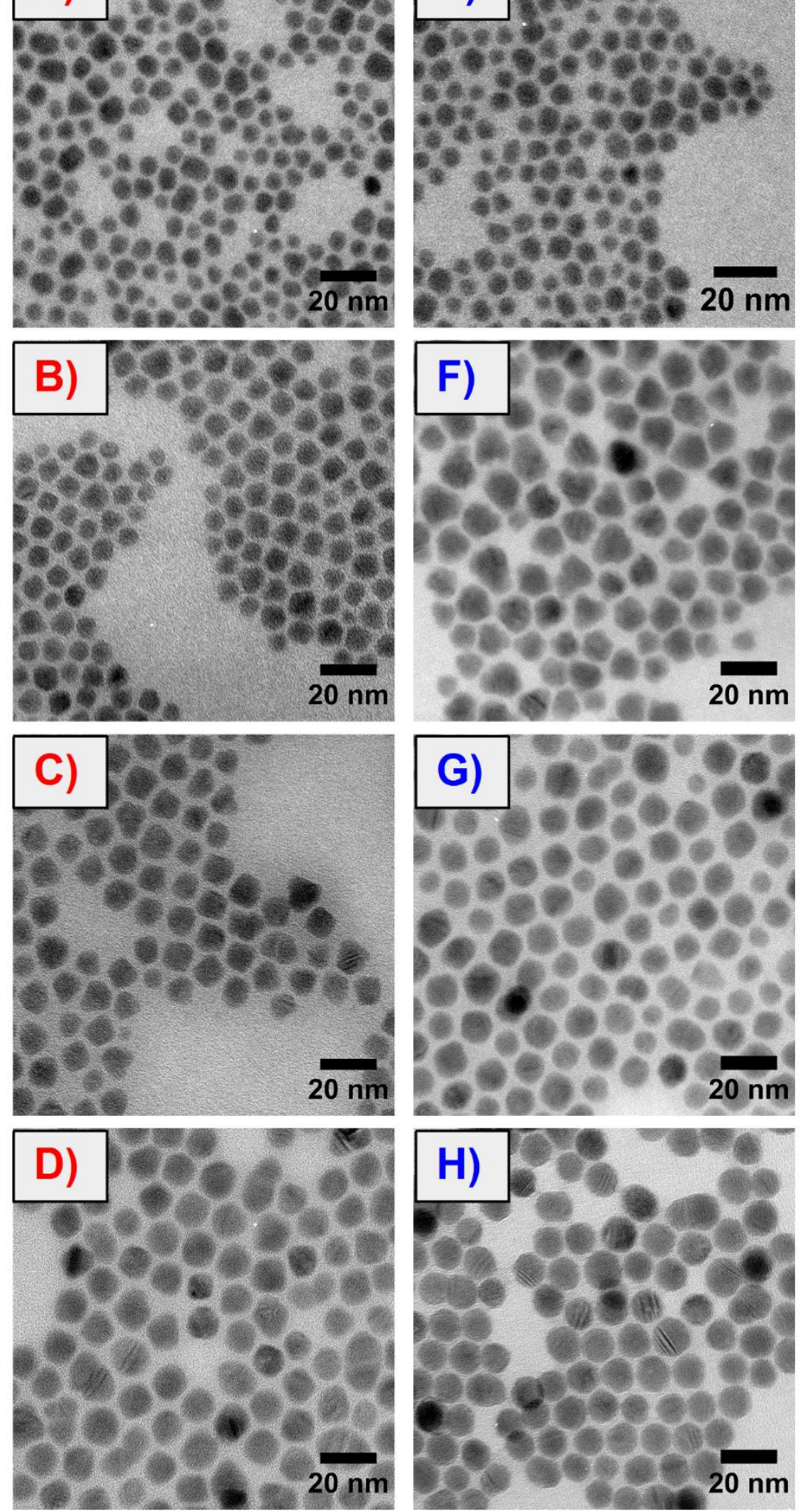
Figure S1. TEM images of a) CdSe/CdS QDs of $5.0 \pm 0.9 \mathrm{~nm}, \mathrm{~b}) 8.5 \pm 1.2 \mathrm{~nm}$, c) $9.9 \pm 1.6 \mathrm{~nm}$ and d) $13.4 \pm 2.0 \mathrm{~nm}$ produced from the same $3 \mathrm{~nm}$ CdSe cores via the flash synthesis. TEM images of e) CdSe/CdS/ZnS QDs of $6.8 \pm 1.0 \mathrm{~nm}, \mathrm{f}) 10.9 \pm 1.5 \mathrm{~nm}, \mathrm{~g}$ ) 11.3 $\pm 1.3 \mathrm{~nm}$ and h) $14.0 \pm 1.5 \mathrm{~nm}$ grown from a), b), c) and d) respectively.

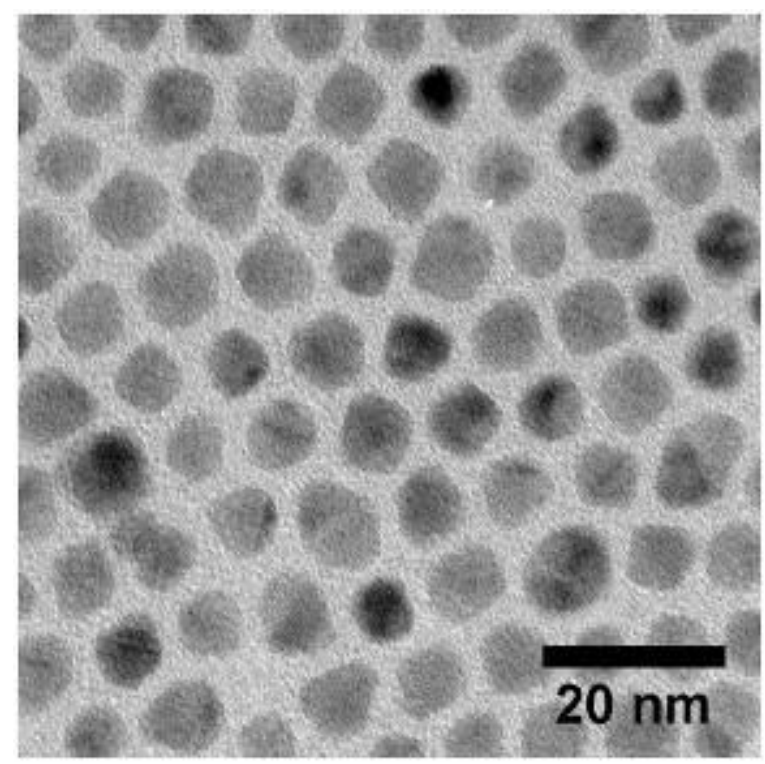

Figure S2. TEM images of core-shell CdSe/CdS QDs with size dispersions below 10\% when increasing the concentration of the seeds from $200 \mathrm{nmol}$ to $400 \mathrm{nmol}$ in the flash synthesis.

Table S1. Overview of properties of core-shell QDs with different sizes.

\begin{tabular}{llllccc}
\hline Sample & Seeds used & \multicolumn{1}{c}{ Composition } & Diameter $(\mathrm{nm})$ & ZnS thickness (nm) & Emission (nm) & PLQY (\%) \\
\hline QD 2 & 3 nm CdSe & CdSe/CdS & $5.0 \pm 0.9$ & - & 586 & 48 \\
QD 3 & QD 2 & CdSe/CdS/ZnS & $6.8 \pm 1.0$ & 0.9 & 587 & 51 \\
QD 4 & 3 nm CdSe & CdSe/CdS & $8.5 \pm 1.2$ & - & 615 & 67 \\
QD 5 & QD 4 & CdSe/CdS/ZnS & $10.9 \pm 1.5$ & 1.2 & 614 & 68 \\
QD 6 & $3 \mathrm{~nm} \mathrm{CdSe}$ & CdSe/CdS & $9.9 \pm 1.6$ & - & 622 & 60 \\
QD 7 & QD 6 & CdSe/CdS/ZnS & $11.3 \pm 1.3$ & 0.7 & 617 & 72 \\
QD 8 & $3 \mathrm{~nm} \mathrm{CdSe}$ & $\mathrm{CdSe} / \mathrm{CdS}$ & $13.4 \pm 2.0$ & - & 622 & 36 \\
QD 9 & QD 8 & $\mathrm{CdSe} / \mathrm{CdS} / \mathrm{ZnS}$ & $14.0 \pm 1.5$ & 0.3 & 620 & 47 \\
\hline
\end{tabular}



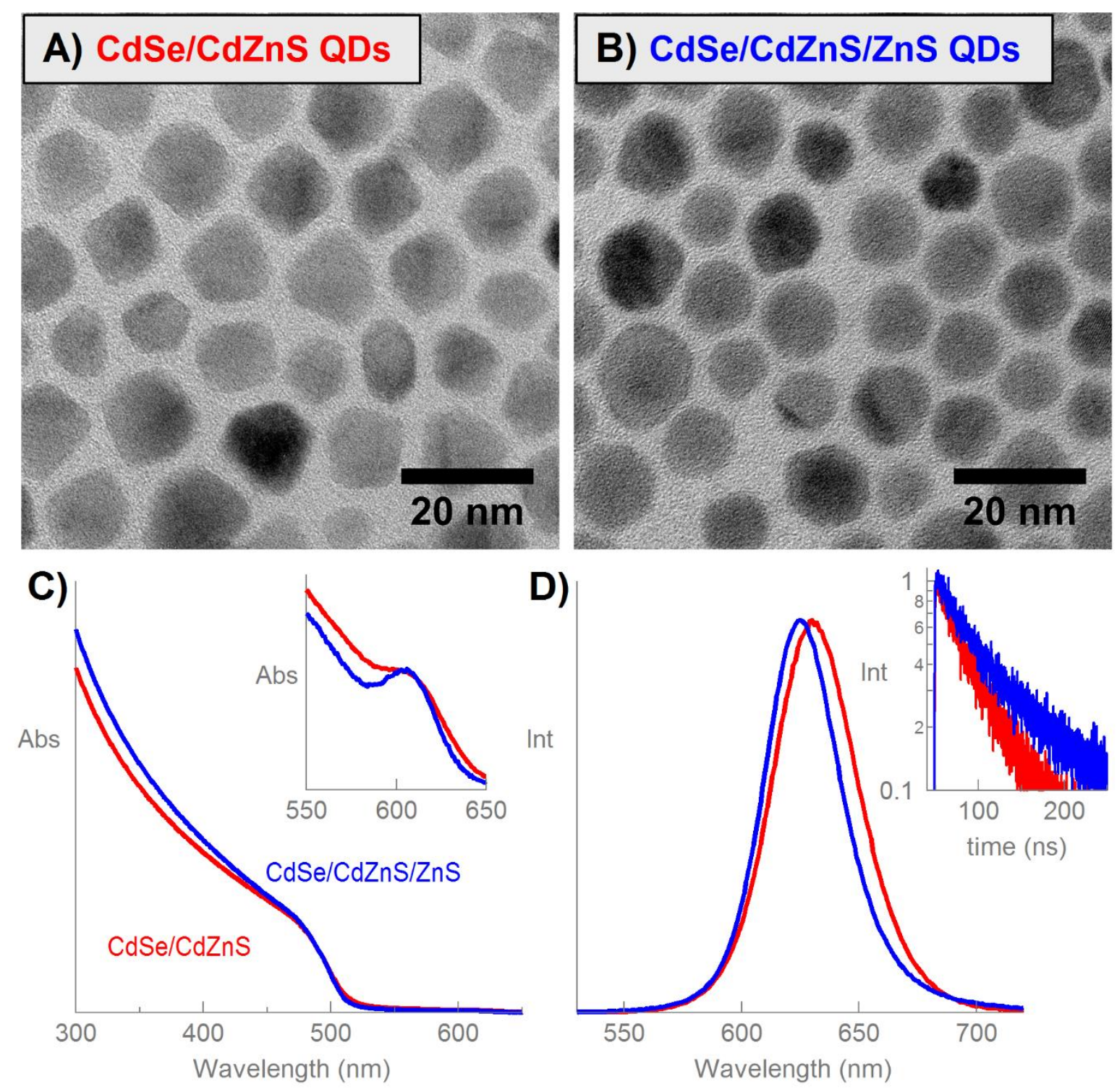

Figure S3. TEM images of a) CdSe/CdZnS and b) CdSe/CdZnS/ZnS QDs. Optical properties with c) normalized absorption spectra (inset: focus on the excitonic feature at $600 \mathrm{~nm}$ ), d) normalized emission spectra (inset: normalized photoluminescence decays of the QDs). 

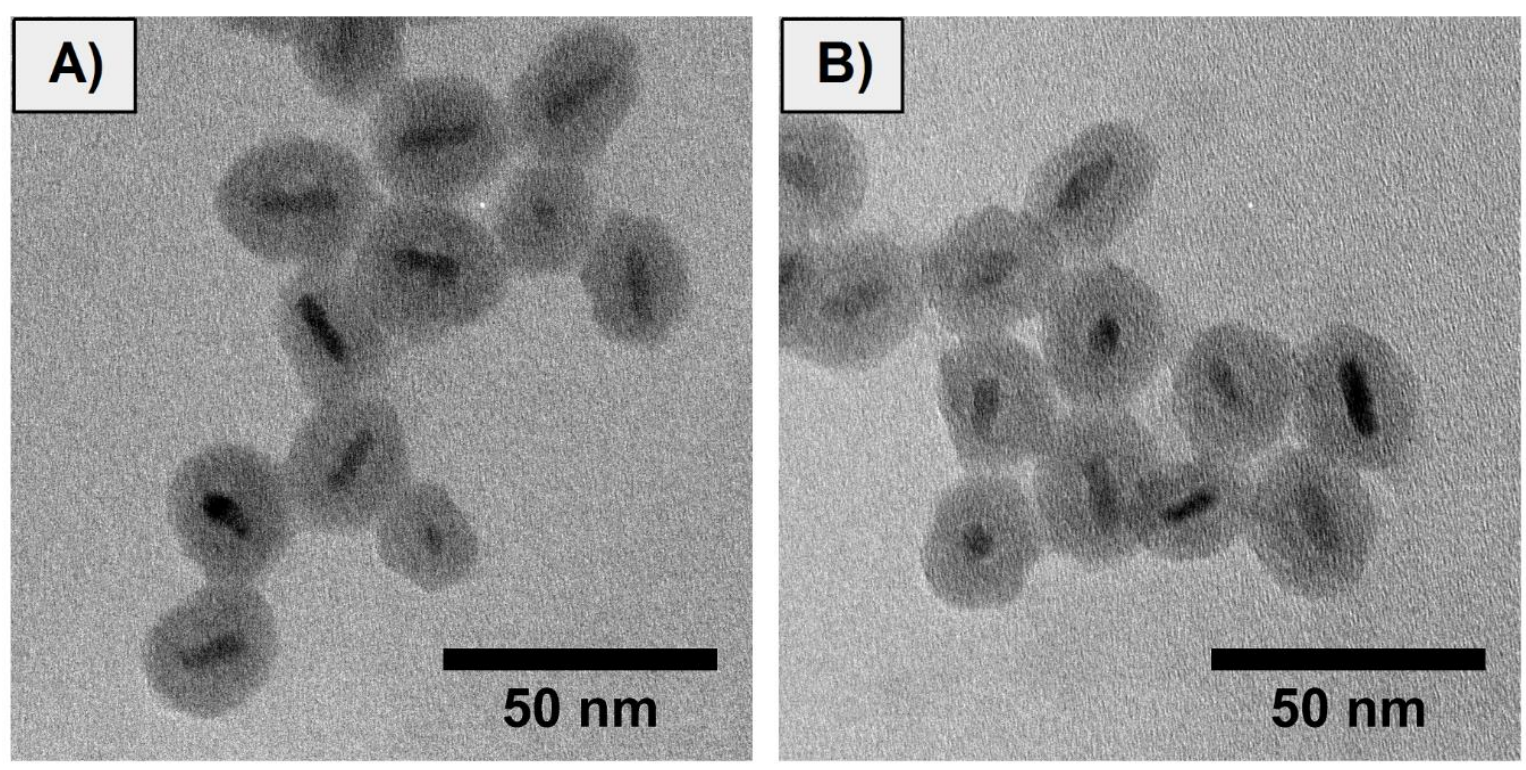

Figure S4. TEM images of a) CdSe/CdS NRs and b) CdSe/CdS/ZnS NRs encapsulated in a silica matrix which display no tip-to-tip connections. The NRs were encapsulated in a silica matrix through a water-in-oil microemulsion process according to a procedure described in the literature. ${ }^{15}$ Briefly, $0.2 \mathrm{nmol}$ of NRs was mixed to $2 \mathrm{~mL}$ of heptane and $640 \mu \mathrm{L}$ of surfactant (Brij30). After 15 minutes of stirring $110 \mu \mathrm{L}$ of $3 \%$ ammonia solution in Milli-Q water was added to the mixture. After 1 hour of stirring, $25 \mu \mathrm{L}$ of tetraethyl orthosilicate (TEOS) was added. The reaction was stopped after 3 day by adding $2 \mathrm{~mL}$ of EtOH in order to destabilize the microemulsion. The nanoparticles were collected by centrifugation and purified by repeated centrifugation in EtOH and two times in Milli-Q water. The particles were stored in Milli-Q water.

\section{TOC graphic}

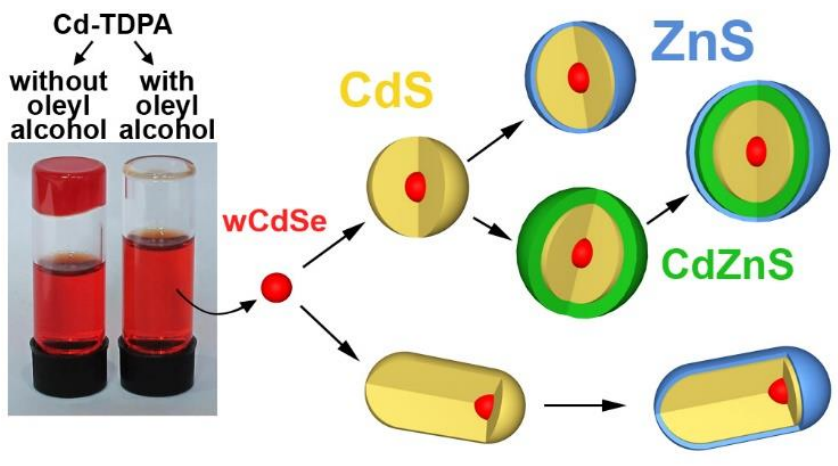

